Article

\title{
Ventilation Heat Recovery from Wood-Burning Domestic Flues. A Theoretical Analysis Based on a Triple Concentric Tube Heat Exchanger
}

\author{
Pierre Peigné $^{1}$, Christian Inard ${ }^{2, *}$ and Lionel Druette ${ }^{1}$
}

1 CERIC Laboratory (Centre d'Essais et de Recherche des Industries de la Cheminée), Poujoulat Group, 79270 Saint-Symphorien, France; E-Mails: p.peigne@laboratoire-ceric.com (P.P.); 1.druette@laboratoire-ceric.com (L.D.)

2 LaSIE (Laboratoire des Sciences de l'Ingénieur pour l'Environnement), Department of Civil Engineering, University of La Rochelle, 17042 La Rochelle, France

* Author to whom correspondence should be addressed; E-Mail: christian.inard@univ-lr.fr; Tel.: +33-(0)546457246; Fax: +33-(0)546458241.

Received: 9 November 2012; in revised form: 24 December 2012 / Accepted: 5 January 2013 / Published: 15 January 2013

\begin{abstract}
This paper presents a new air-heating system concept for energy-efficient dwellings. It is a system designed to heat a low-energy building by coupling a heat-recovery ventilation system with a three-fluid heat exchanger located on the chimney of a wood-pellet stove. The proposed work focuses on the heat transfer that occurs between flue gases, the ventilation air and the combustion air within a triple concentric tube heat exchanger with no insulation at its outer surface. The main objective is to predict outlet temperature for the specific geometry of the heat exchanger studied here. Thus, the governing differential equations are derived for a counter-co-current flow arrangement of the three fluids. Then analytical solutions for the steady-state temperature distribution are obtained as well as the amount of heat transferred to the outside. An expression for the effectiveness of the heat exchanger is also proposed. Based on these results, calculations are performed on a case study to predict the fluid temperature distribution along the heat exchanger. Finally, a parametric study is carried out on this case study to assess the influence of the relevant parameters on the effectiveness of the heat exchanger. In addition, computation of heat losses to the outside justifies whether insulation is needed.
\end{abstract}


Keywords: low energy building; air heating system; wood pellet stove; heat recovery ventilation; triple concentric tube heat exchanger; analytical solution

\section{Nomenclature:}

$A \quad$ heat transfer area $\left(\mathrm{m}^{2}\right)$;

$c_{p} \quad$ specific heat capacity at constant pressure $[\mathrm{J} /(\mathrm{kg} \cdot \mathrm{K})]$;

$C$ heat capacity rate $(\mathrm{J} / \mathrm{K})$;

$C_{r} \quad$ dimensionless heat capacity ratio (-);

$d A \quad$ differential heat transfer area $\left(\mathrm{m}^{2}\right)$;

$d \phi \quad$ differential heat flow rate $(\mathrm{W})$;

$d x \quad$ axial discretization step $(\mathrm{m})$;

$D$ diameter (m);

$D_{h} \quad$ hydraulic diameter (m);

e thickness (m);

E effectiveness (\%);

$h \quad$ convective heat transfer coefficient $\left[\mathrm{W} /\left(\mathrm{m}^{2} \cdot \mathrm{K}\right)\right]$;

$h_{r} \quad$ radiant heat transfer coefficient $\left(\mathrm{W} /\left(\mathrm{m}^{2} \mathrm{~K}\right)\right)$;

$h_{w} \quad$ conductive heat transfer coefficient $\left[\mathrm{W} /\left(\mathrm{m}^{2} \cdot \mathrm{K}\right)\right]$;

\section{Greek Symbols}

$\varepsilon \quad$ emissivity (-);

$\lambda \quad$ thermal conductivity $[\mathrm{W} /(\mathrm{m} \cdot \mathrm{K})]$;

$\sigma \quad$ Stephan-Boltzmann constant $\left[5.67 \times 10^{-8} \mathrm{~W} /\left(\mathrm{m}^{2} \cdot \mathrm{K}^{4}\right)\right]$;

$\phi \quad$ heat flow rate $(\mathrm{W})$;

$\eta_{f} \quad$ fin efficiency (-);

\section{Subscripts}

0 ambient air (surroundings);

1 first fluid (flue gases);

2 second fluid (ventilation air);

3 third fluid (combustion air);

$a$ internal tube;

$b$ intermediate tube;

c external tube;

$o$ outside (surroundings);

$f \quad$ fin(s);

in inlet;
$L \quad$ length (m);

$\dot{m}$ mass flow rate $(\mathrm{kg} / \mathrm{s})$;

$N$ dimensionless ratio of overall conductance $U A$ and heat capacity rate $C(-)$;

$\mathrm{Nu}$ Nusselt number (-);

NTU number of transfer units (-);

$P \quad$ perimeter (m);

Pr Prandtl number (-);

$r \quad$ radius (m);

$R a \quad$ Rayleigh number (-);

Re Reynolds number (-);

$T$ temperature (K);

$U$ overall heat transfer coefficient

$$
\left[\mathrm{W} /\left(\mathrm{m}^{2} \cdot \mathrm{K}\right)\right] \text {; }
$$

$X \quad$ dimensionless length coordinate (-);

$x \quad$ axial coordinate $(\mathrm{m})$;

$z \quad$ heat capacity rate ratio $(\min / \max )(-)$.

$\eta_{o} \quad$ overall surface efficiency (-);

$\theta \quad$ dimensionless temperature (-);

$\Delta T$ temperature difference $(\mathrm{K})$;

$\Delta T_{\text {in }}$ temperature difference depending on inlet temperatures and heat capacity rates $(\mathrm{K})$.

out outlet;

ext external;

int internal;

inner inner;

outer outer;

actual actual;

$m$ mean;

max maximum;

min minimum;

tot total. 
Acronyms

\begin{tabular}{|c|c|c|c|}
\hline $\begin{array}{l}\text { FG } \\
\text { VA }\end{array}$ & $\begin{array}{l}\text { flue gases; } \\
\text { ventilation air; }\end{array}$ & TCTHE & $\begin{array}{l}\text { triple concentric tube heat } \\
\text { exchanger; }\end{array}$ \\
\hline CA & combustion air; & TCTHE-PI & triple concentric tube heat \\
\hline OUT & outside; & & exchanger with perfect insulation \\
\hline TOT & total; & TCTHE-NI & triple concentric tube heat \\
\hline RSWPS & room-sealed wood pellet stov & & exchanger with no insulation; \\
\hline MVHR & $\begin{array}{l}\text { mechanical ventilation heat } \\
\text { recovery; }\end{array}$ & DCTHE-NI & $\begin{array}{l}\text { double concentric tube heat } \\
\text { exchanger with no insulation. }\end{array}$ \\
\hline
\end{tabular}

\section{Introduction}

Low energy buildings are characterized by a suitable orientation, a high thermal insulation and a very low air leakage [1]. One of the first results is that air-to-air heat recovery systems are increasingly integrated into the ventilation systems of buildings. In this context, Mardiana-Idayu and Riffat [2] have carried out a broad review on heat recovery technologies for building applications. First this study presents various types of heat recovery used in buildings namely fixed-plate, heat pipe, rotary wheel and run-around units. Then, the performance of heat recovery integrated in the ventilation systems of buildings including the coupling with dehumidification systems as well as the use of solar energy is presented and discussed. It should be noticed that the proposed system in this study is not mentioned in the review showing its innovative side. In addition, many studies focus on the efficiency of ventilation systems with heat recovery compared to standard ventilation systems $[3,4]$. Generally speaking and for the moderate climate region of middle Europe which is discussed in this study, it is shown that the use of heat recovery on air ventilation is not always competitive when operating costs are taken into account. Thus, the use of low power fans and low pressure loss systems is strongly recommended. Moreover, for very low energy buildings, traditional heating systems are no longer required nor even suited to these constructions, as highlighted by Feist [5]. In this context, heating requirements are low enough to be provided by the ventilation, with fresh air flow rates only and a maximum supply air temperature of about $50{ }^{\circ} \mathrm{C}$. In such dwellings, a balanced mechanical ventilation system with a high efficiency heat recovery is especially recommended and the remaining heat demand could be easily covered by a renewable energy source, as a wood stove for example $[5,6]$.

However, wood burning appliances present the disadvantage of concentrating heat only in the room where they are installed. Therefore, systems mechanically extracting hot air around the device are often used to redistribute heat to other rooms in the house. Nevertheless, the Consumer Safety Commission [7] pointed out that this type of system could create a negatively pressurised zone around the heating appliance which often leads to dust diffusion, but even can cause flue gases to be partially drawn in and redistributed in the other rooms. As a consequence, polluted air with abnormally high levels of carbon monoxide could place occupants at risk.

Instead of taking hot air above the heating appliance, as do conventional systems, the combined system presented in this study recovers heat by blowing fresh air into a specific heat exchanger, which is both integrated into the chimney of a wood pellet stove and connected to the ventilation air supply 
network. Hence, while ensuring occupant safety, this combined system aims to meet the requirements of both heating and indoor air quality in energy efficient dwellings. A detailed description of this combined system comprising of a room-sealed wood pellet stove (RSWPS), a mechanical ventilation heat recovery (MVHR) and a triple concentric tube heat exchanger (TCTHE), is given in [8].

Considering that ideal configuration should combine all the advantages of the RSWPS and the MVHR while also introducing the TCTHE to better distribute heat in the house, the authors recommend coupling the three main components of the combined system as it is shown in Figure 1. As discussed in details in [8], this is a relevant configuration to standardize temperatures in the best way throughout the house and prevent any risk of ventilation air contamination by the combustion products. It should be noticed that the part of fresh air circulating in the TCTHE is called ventilation air.

The main objective of this work is to develop a theoretical model of a triple concentric tube heat exchanger in order to compute steady-state temperature distribution of the three fluids namely flue gases, ventilation air and combustion air. This will provide heat fluxes and effectiveness of the heat exchanger.

Figure 1. Scheme of the combined system configuration.

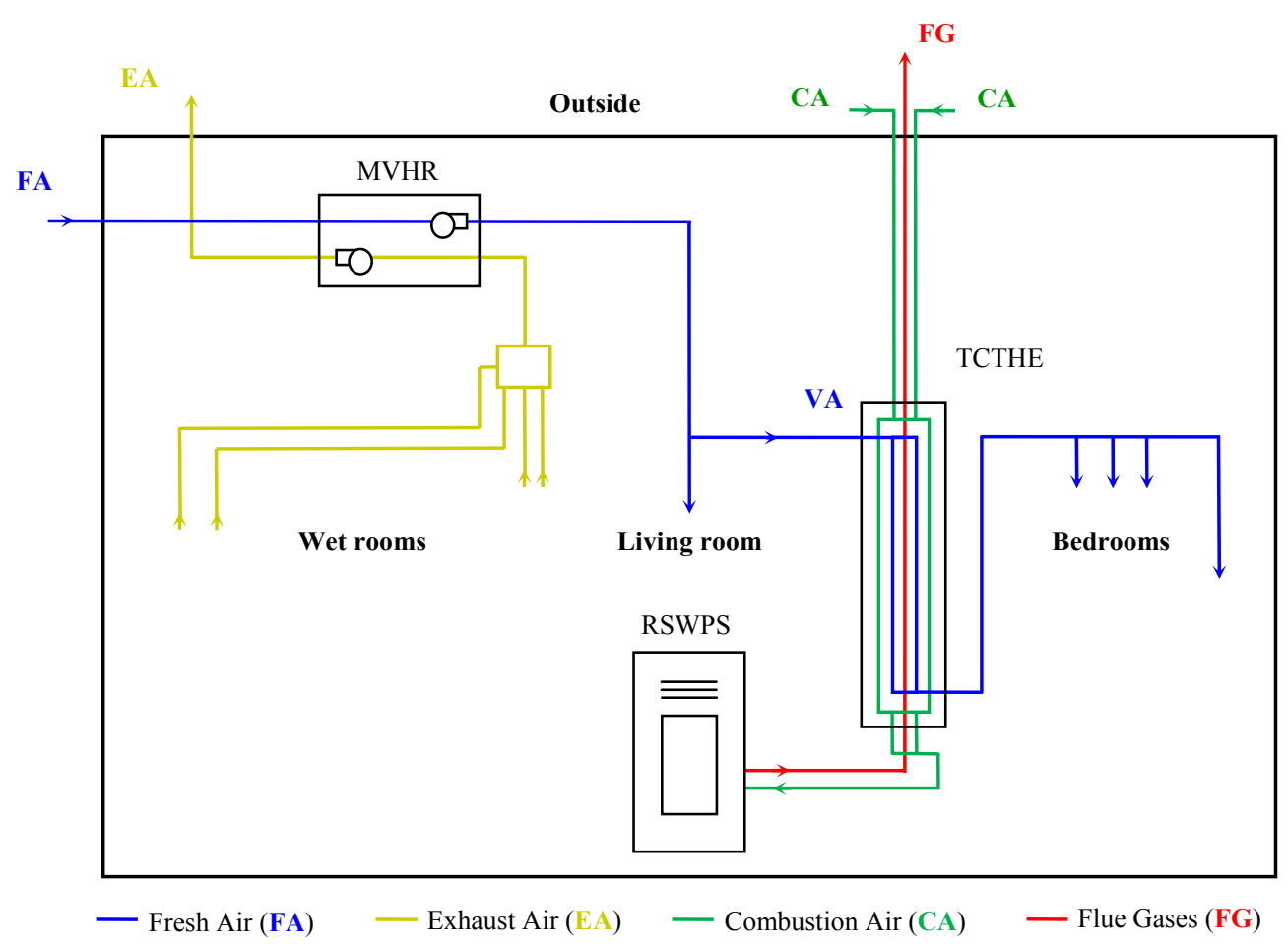

As described on the diagram in Figure 2, three different fluids are involved in heat transfers that occur in the triple concentric tube heat exchanger which has been developed in this study. Indeed, the flue gases (FG) are evacuated through the inner tube, the ventilation air (VA) recovers heat by circulating in the first annulus between the inner tube and the intermediate tube, and the combustion air (CA) which ensures the correct operation of the wood pellet stove circulates from top to bottom in the second annulus between the intermediate tube and the outer tube. In that way, flue gases and ventilation air are in a counter-flow arrangement, while ventilation air and combustion air are in a parallel-flow arrangement. 
Figure 2. Diagram of the TCTHE.

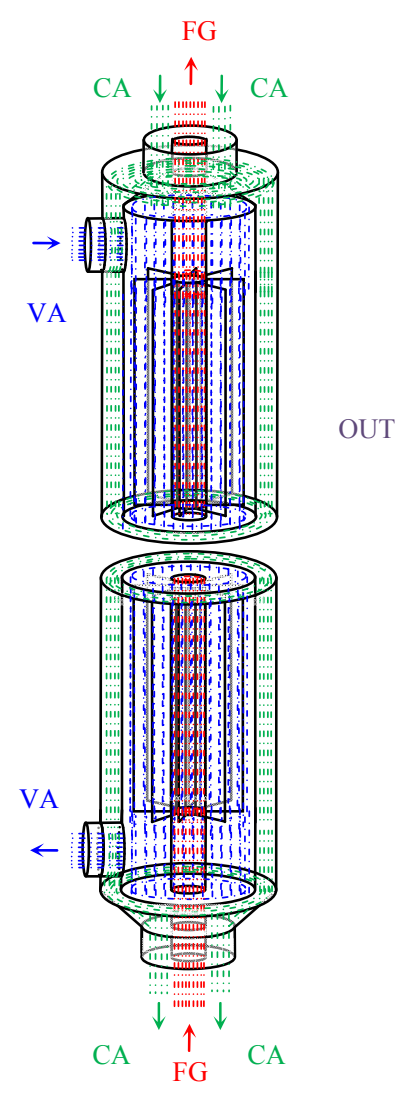

\section{Theoretical Analysis}

After the first analysis performed by Morley [9], many studies [10-12] have been conducted on exchanges involving three fluids in parallel stream flow arrangements and steady-state operation. For example, a significant design theory has been developed by Sorlie [13] for two parallel stream arrangements of a three-fluid heat exchanger with two thermal communications. Then, Zuritz [14] developed a set of analytical equations, he solved using Laplace transformation, for the computation of temperature distributions in a triple concentric tube heat exchanger. In other various cases studied [15-19], two or three thermal communications are considered, as well as four different flow arrangements and important dimensionless parameters. Thus, an extensive review of thermal design theory and steady state performance of three-fluid heat exchangers has been established by Sekulic and Shah [20] in order to unify the approach and systematize the solutions. To the knowledge of the authors, there has been little investigation of heat transfers in a triple concentric tube heat exchanger with no insulation at the outer surface (TCTHE-NI). In that way, this work is more related to studies by Prasad [21,22] on double concentric tube heat exchangers with no insulation at the outer surface (DCTHE-NI), and by Ünal [23-25] on triple concentric tube heat exchangers with perfect insulation at the outer surface (TCTHE-PI).

As depicted in Figure 3, the physical model of the TCTHE-NI consists of three concentric tubes forming one circular flow passage and two concentric annular flow passages. Flue gases (FG), called fluid 1 , enters the inner tube at $x=L$ and flows in negative $x$-direction, while ventilation air (VA) and combustion air (CA), called fluids 2 and 3, respectively, both enter the heat exchanger at $x=0$ and 
flow in both annulus section in positive $x$-direction. Not to mention heat transfers with ambient air on the surroundings of the heat exchanger, because there is no insulation at its outer surface (OUT).

The theoretical analysis of the TCTHE-NI in a specific combination of counter-co-current flow arrangement, as defined in [20], has been carried out with the following assumptions:

- The system operates under steady-state conditions;

- Phase change does not take place;

- Bulk mean temperatures only depend on axial $(x)$ direction;

- The thermo-physical properties of the fluid streams are constant and uniform;

- Thermal conduction is not assumed in fluids or walls parallel to the fluid flow direction;

- Heat transfer coefficients are independent of time and axial position;

- Temperature of the outside remains constant.

Figure 3. Physical model for the TCTHE-NI.

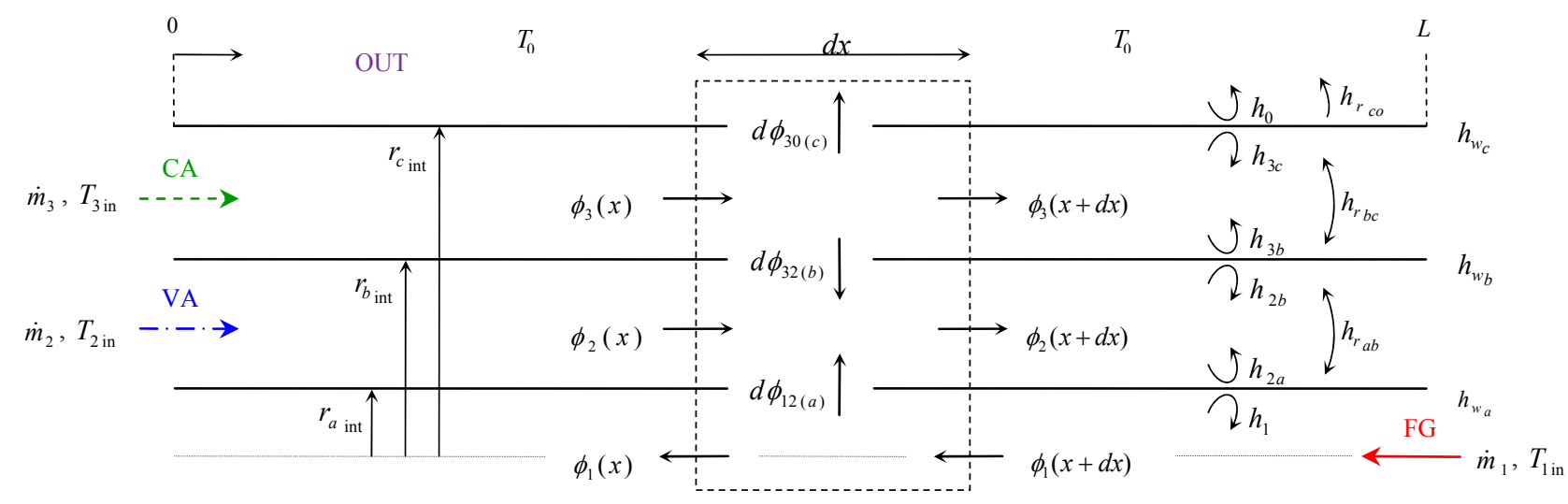

\subsection{Mathematical Model}

Under the above assumptions, the energy balance on a control volume of length $d x$ yields the following set of equations:

$$
\begin{gathered}
d \phi_{1}=d \phi_{12(a)} \\
d \phi_{2}=d \phi_{12(a)}+d \phi_{32(b)} \\
d \phi_{3}=-d \phi_{32(b)}-d \phi_{30(c)}
\end{gathered}
$$

where the indices $0,1,2$ and 3 denote outside, flue gases in the inner tube, ventilation air in the first annulus and combustion air in the second annulus, respectively.

In Equations (1) and (3), the differential heat flow rates $d \phi_{1}$ and $d \phi_{3}$ represent the heat lost by hot fluids 1 and 3 between locations $x$ and $x+d x$. In Equation (2), the differential heat flow rate $d \phi_{2}$ denotes the heat retrieved by the cold fluid 2 between locations $x$ and $x+d x$. Since there is no phase change in any fluids, the differential heat flow rates $d \phi$ can be expressed in terms of mass flow rates $\dot{m}$ specific heats $c_{p}$, and temperature differentials $d T$ :

$$
\forall n \in\{1,2,3\} \quad d \phi_{n}=\phi_{n}(x+d x)-\phi_{n}(x)=\left(\dot{m} c_{p}\right)_{n} d T_{n}=C_{n} d T_{n}
$$


In the meantime and on the same differential control volume, the differential heat flow rates $d \phi_{i j(k)}$ transferred from a hot fluid $i$ to a cold fluid $j$ through a tube wall $k$ can be expressed in terms of the difference between bulk temperatures $T_{i}-T_{j}$, the value of the overall heat transfer coefficients $U_{k}$ and the corresponding differential heat transfer areas $d A_{k}$ :

$$
d \phi_{i j(k)}=U_{k} d A_{k}\left(T_{i}-T_{j}\right)
$$

with: $\forall k \in\{a, b, c\}$

$$
d A_{k}=2 \pi r_{k} d x=\pi D_{k} d x=P_{k} d x
$$

According to an electrical analogy, the thermal circuit presented in Figure 4 is used to describe the combined heat transfers in the TCTHE-NI. The conduction, convection and radiation resistances per unit area, called $1 / h_{w}, 1 / h$ and $1 / h_{r}$ respectively, are computed from Equations (11)-(13).

Figure 4. Electrical analogy of the combined modes of heat transfer in the TCTHE-NI.

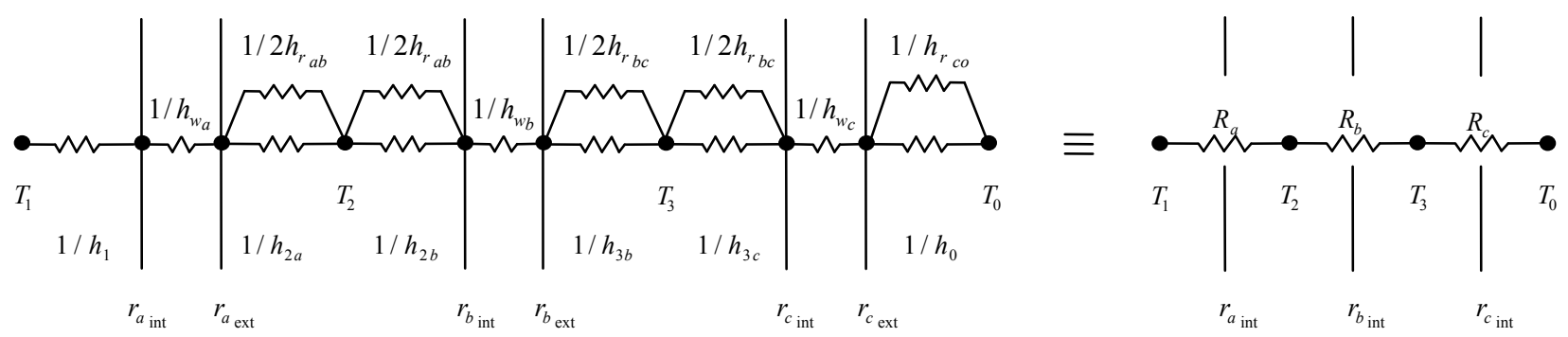

Concerning radiation, it should be noticed that flue gases, ventilation air and combustion air are non-participating fluids. Here, while maintaining the same radiant thermal resistance, the expression of the heat transfer by radiation in the annuli is assumed to be split in two parts involving the bulk temperature of the fluid between tube walls. In this way, the thermal resistance $R_{k}$ of the tube $k$, or the overall heat transfer coefficient $U_{k}$ which is arbitrary defined here in terms of the internal area of the tube wall $A_{k \text { int }}=2 \pi r_{k \text { int }} L$, may be expressed for each tube wall as:

$$
\begin{gathered}
R_{a}=\frac{1}{U_{a} A_{a_{\mathrm{int}}}}=\frac{1}{h_{1} A_{a_{\mathrm{int}}}}+\frac{1}{h_{w_{a}} A_{a_{\mathrm{int}}}}+\frac{1}{\left(h_{2 a}+2 h_{r_{a b}}\right) A_{a_{\text {int }}}}\left(\frac{r_{a_{\text {int }}}}{r_{a_{\mathrm{ext}}}}\right) \\
R_{b}=\frac{1}{U_{b} A_{b_{\mathrm{int}}}}=\frac{1}{\left(h_{2 b}+2 h_{r_{a b}}\right) A_{b_{\text {int }}}}+\frac{1}{h_{w_{b}} A_{b_{\text {int }}}}+\frac{1}{\left(h_{3 b}+2 h_{r_{b c}}\right) A_{b_{\text {int }}}}\left(\frac{r_{b_{\text {int }}}}{r_{b_{\text {ext }}}}\right) \\
R_{c}=\frac{1}{U_{c} A_{c_{\text {int }}}}=\frac{1}{\left(h_{3 c}+2 h_{r_{b c}}\right) A_{c_{\text {int }}}}+\frac{1}{h_{w_{c}} A_{c_{\text {int }}}}+\frac{1}{\left(h_{0}+h_{r_{c o}}\right) A_{c_{\text {int }}}}\left(\frac{r_{c_{\text {int }}}}{r_{c_{\text {ext }}}}\right)
\end{gathered}
$$

Taking the example of this study where fins are added on the external wall of the inner tube $a$ to enhance the heat recovered by the ventilation air, the overall surface efficiency $\eta_{o}$ should be introduced. Thus, the convective heat transfer coefficient of the external wall of the inner tube $h_{2 a}$ should be replaced in Equation (7) by $h_{2 a f}$ :

$$
h_{2 a f}=\eta_{o} \frac{A_{t o t}}{A_{a_{\mathrm{int}}}} h_{2 a}
$$


with: $\eta_{o}=1-\frac{\mathrm{nb}_{f} A_{f}}{A_{\text {tot }}}\left(1-\eta_{f}\right)$.

where $\mathrm{nb}_{f}$ is the number of fins, $A_{f}$ is the surface area of one fin, $A_{t o t}$ is the total surface area i.e., both the fins and the exposed portion of the base, and $\eta_{f}$ is the efficiency of one fin. Expressions for the fin efficiency $\eta_{f}$ are available in the literature [26] for several common fin arrays.

Then, the following expressions are used to assess the various coefficients that describe heat transfers by conduction, convection and radiation.

- Conductive heat transfer coefficient

Through a cylindrical wall $k$, the thermal resistance for conduction $R_{w k}$, or the radial conductive heat transfer coefficient $h_{w k}$ which is defined with respect to the internal wall surface area $A_{k i n t}$, may be expressed in terms of thermal conductivity $\lambda_{k}$, radius ratio $r_{k \text { ext }} / r_{k \text { int }}$ and heat exchanger length $L$ :

$$
\forall k \in\{a, b, c\} \quad R_{w_{k}}=\frac{1}{h_{w_{k}} A_{k}}=\frac{\ln \left(r_{k_{\mathrm{ext}}} / r_{k_{\text {int }}}\right)}{2 \pi \lambda_{k} L}
$$

- Convective heat transfer coefficient

The convective heat transfer coefficient $h_{i}$ of the fluid $i$ is calculated using Nusselt number $N u$, fluid thermal conductivity $\lambda_{i}$ and hydraulic diameter $D_{h}$ :

$$
\forall i \in\{1,2,3,0\} \quad h_{i}=N u \lambda_{i} / D_{h}
$$

where $D_{h}=D_{\text {outer }}-D_{\text {inner }}$ for an annular space between two (inner and outer) cylindrical walls. Then the average convection coefficients are determined thanks to correlations from the literature.

For forced convection laminar flow $(R e \leq 2300)$, Sieder and Tate [26] correlation is used both for the inner tube and for the annulus:

$$
N u=1.86\left(\operatorname{Re} \operatorname{Pr} D_{h} / L\right)^{1 / 3}
$$

For forced convection transition and turbulent flows $(R e>2300)$, Colburn [26] correlation is used for the inner tube and Monrad and Pelton (cited by Jakob [27] and by Garcia-Valladares [28]) correlations are used for the annulus.

Inner tube:

$$
\begin{array}{ll}
N u=0.023 \operatorname{Re}^{4 / 5} \operatorname{Pr}^{1 / 3} & \\
N u=0.020 \operatorname{Re}^{4 / 5} \operatorname{Pr}^{1 / 3}\left(D_{\text {outer }} / D_{\text {inner }}\right)^{0,53} & \text { for the inner wall } \\
N u=0.027 \operatorname{Re}^{4 / 5} \operatorname{Pr}^{1 / 3} & \text { for the outer wall }
\end{array}
$$

Annulus:

For free convection, the following correlations [26] are used to evaluate the heat transfer coefficient at the vertical external surface of the heat exchanger:
Laminar flow:
$10^{4} \leq R a \leq 10^{9}$
$N u=0.59 R a^{1 / 4}$
Turbulent flow: $\quad 10^{9} \leq R a \leq 10^{13}$
$N u=0.10 R a^{1 / 3}$

According to the three flow rates and the three tubes diameters studied here and presented in Section 3.1, Reynolds number is always lower than 2300 for the combustion air, whereas it is always higher than 2300 (with a maximum value of 5500) for the flue gases and the ventilation air depending on both operating modes of the wood pellet stove and the ventilation system. Hence, Sieder and Tate 
correlation, Colburn correlation and Monrad and Pelton correlation are used respectively for combustion air, flue gases and ventilation air.

- Radiant heat transfer coefficient

The radiant heat transfer coefficient $h_{r k l}$ between two diffuse gray surfaces $k$ and $l$ that form an enclosure can be expressed as [26]:

$$
\forall(k l) \in\{(a b) ;(b c) ;(c o)\} \quad h_{r_{k l}}=\sigma F_{k l}\left(T_{k}^{4}-T_{l}^{4}\right) \approx \sigma F_{k l} 4 T_{\mathrm{m} k l}^{3}
$$

with: $\quad F_{a b}=\left[\frac{1}{\varepsilon_{a}}+\frac{1-\varepsilon_{b}}{\varepsilon_{b}}\left(\frac{r_{a_{\text {ext }}}}{r_{b_{\text {int }}}}\right)\right]^{-1} \quad F_{b c}=\left[\frac{1}{\varepsilon_{b}}+\frac{1-\varepsilon_{c}}{\varepsilon_{c}}\left(\frac{r_{b_{\text {ext }}}}{r_{c_{\text {int }}}}\right)\right]^{-1} \quad F_{c o}=\varepsilon_{c}$

and: $T_{\mathrm{m} a b} \approx \frac{T_{\mathrm{m} 1}+T_{\mathrm{m} 2}}{2} T_{\mathrm{m} b c} \approx \frac{T_{\mathrm{m} 2}+T_{\mathrm{m} 3}}{2} T_{\mathrm{m} c o} \approx \frac{T_{\mathrm{m} 3}+T_{0}}{2}$ as first approximation, and where $\forall i \in\{1,2,3\}$ the mean bulk temperature $T_{\mathrm{m} i}=\frac{T_{i \mathrm{in}}+T_{i \text { out }}}{2}$ is iteratively calculated.

Then, considering the differential surface areas and the overall heat transfer coefficients given by Equations (6)-(9), the set of governing Equations (1)-(3) can thus be written in the following form:

$$
\begin{gathered}
C_{1} d T_{1}=U_{a} d A_{a}\left(T_{1}-T_{2}\right) \\
C_{2} d T_{2}=U_{a} d A_{a}\left(T_{1}-T_{2}\right)+U_{b} d A_{b}\left(T_{3}-T_{2}\right) \\
C_{3} d T_{3}=-U_{b} d A_{b}\left(T_{3}-T_{2}\right)-U_{c} d A_{c}\left(T_{3}-T_{0}\right)
\end{gathered}
$$

To provide a formulation with non-dimensional parameters and for brevity, following definitions are used:

$$
\forall k \in\{a, b, c\} \quad A_{k}=A_{k \text { int }} \quad \text { and } \quad \Delta T_{\text {in }}=\frac{C_{1}\left(T_{1 \text { in }}-T_{2 \text { in }}\right)+C_{3}\left(T_{3 \text { in }}-T_{2 \text { in }}\right)}{C_{1}+C_{3}}
$$

as well as the below non dimensional parameters:

$$
\begin{array}{clc}
X=\frac{x}{L} & C_{r 1}=\frac{C_{1}}{C_{2}} & C_{r 3}=\frac{C_{3}}{C_{2}} \\
N_{1}=\frac{U_{a} A_{a}}{C_{1}} & N_{3}=\frac{U_{b} A_{b}}{C_{3}} & N_{0}=\frac{U_{c} A_{c}}{C_{3}} \\
\theta_{1}=\frac{T_{1}-T_{0}}{\Delta T_{\text {in }}} & \theta_{2}=\frac{T_{2}-T_{0}}{\Delta T_{\text {in }}} & \theta_{3}=\frac{T_{3}-T_{0}}{\Delta T_{\text {in }}}
\end{array}
$$

Hence, the set of governing equations can be written in the form:

$$
\begin{gathered}
\frac{d \theta_{1}}{d X}=N_{1} \theta_{1}-N_{1} \theta_{2} \\
\frac{d \theta_{2}}{d X}=N_{1} C_{r 1} \theta_{1}-\left(N_{1} C_{r 1}+N_{3} C_{r 3}\right) \theta_{2}+N_{3} C_{r 3} \theta_{3} \\
\frac{d \theta_{3}}{d X}=N_{3} \theta_{2}-\left(N_{0}+N_{3}\right) \theta_{3}
\end{gathered}
$$


with boundary conditions: $\quad \theta_{1}(1)=\theta_{1 \text { in }} \quad \theta_{2}(0)=\theta_{2 \text { in }} \quad \theta_{3}(0)=\theta_{3 \text { in }}$

\subsection{Analytical Solutions}

By combining Equations (17)-(19), the following set of third order ordinary differential equations is obtained:

$$
\forall n \in\{1,2,3\} \quad \frac{d^{3} \theta_{n}}{d X^{3}}+A \frac{d^{2} \theta_{n}}{d X^{2}}+B \frac{d \theta_{n}}{d X}+C \theta_{n}=0
$$

where the coefficients $A, B$ and $C$ are given by:

$$
A=N_{0}+N_{1}\left(C_{r 1}-1\right)+N_{3}\left(C_{r 3}+1\right), B=N_{1}\left(N_{0}+N_{3}\right)\left(C_{r 1}-1\right)+N_{3} C_{r 3}\left(N_{0}-N_{1}\right), C=-N_{0} N_{1} N_{3} C_{r 3}
$$

General solutions of the above system of 3 linear homogeneous third order ordinary differential Equation (21) are in the form of a sum of exponential functions in $X$ :

$$
\forall n \in\{1,2,3\} \quad \theta_{n}(X)=\alpha_{n} e^{\lambda_{1} X}+\beta_{n} e^{\lambda_{2} X}+\gamma_{n} e^{\lambda_{3} X}
$$

where the coefficients $\alpha_{n}, \beta_{n}$ and $\gamma_{n}$ should be determined for $n=1,2$ and 3 with boundary conditions and where the coefficients $\lambda_{1}, \lambda_{2}$ and $\lambda_{3}$ are solutions of the characteristic Equation (23):

$$
\lambda^{3}+A \lambda^{2}+B \lambda+C=0
$$

Cardano's method is used to solve the cubic Equation (23). Thus, the quadratic term is eliminated and the so-called depressed cubic form is obtained:

$$
x^{3}+p x^{2}+q=0
$$

with $x=\lambda+\frac{A}{3}, p=-\frac{A}{3}+B, q=\frac{A}{27}\left(2 A^{2}-9 B\right)+C$.

Considering the range of variation of the parameters used in this study, the discriminant $\Delta$ of the reduced form is always strictly negative:

$$
\Delta=q^{2}+\frac{4}{27} p^{3}<0
$$

Hence, Equation (24) has three real roots:

$$
\forall n \in\{1,2,3\} \quad x_{n}=2 \sqrt{\frac{-p}{3}} \cos \left[\frac{1}{3} \arccos \left(\frac{-q}{2} \sqrt{\frac{27}{-p^{3}}}\right)+\frac{2 n \pi}{3}\right]
$$

and the three solutions of Equation (23) are:

$$
\forall n \in\{1,2,3\} \quad \lambda_{n}=x_{n}-\frac{A}{3}
$$

Then, using Equations (17)-(19), the boundary conditions given by Equation (20) and the form of the solutions given by Equation (22) yields a set of three equations for each fluid:

$\underline{\text { Fluid } 1}$

$$
\begin{gathered}
\theta_{1}(0)=\alpha_{1}+\beta_{1}+\gamma_{1} \\
\left.\frac{d \theta_{1}}{d X}\right|_{X=0}=\alpha_{1} \lambda_{1}+\beta_{1} \lambda_{2}+\gamma_{1} \lambda_{3}=N_{1}\left[\theta_{1}(0)-\theta_{2}(0)\right]
\end{gathered}
$$




$$
\theta_{1}(1)=\alpha_{1} e^{\lambda_{1}}+\beta_{1} e^{\lambda_{2}}+\gamma_{1} e^{\lambda_{3}}
$$

$\underline{\text { Fluid } 2}$

$$
\theta_{2}(0)=\alpha_{2}+\beta_{2}+\gamma_{2}
$$

$$
\begin{aligned}
& \left.\frac{d \theta_{2}}{d X}\right|_{X=0}=\alpha_{2} \lambda_{1}+\beta_{2} \lambda_{2}+\gamma_{2} \lambda_{3}=N_{1} C_{r 1} \theta_{1}(0)-\left[N_{1} C_{r 1}+N_{3} C_{r 3}\right] \theta_{2}(0)+N_{3} C_{r 3} \theta_{3}(0) \\
& \left.\frac{d \theta_{3}}{d X}\right|_{X=0}=\alpha_{2} \lambda_{1}^{\prime \prime}+\beta_{2} \lambda_{2}^{\prime \prime}+\gamma_{2} \lambda_{3}^{\prime \prime}+K_{0}\left(\alpha_{1}+\beta_{1}+\gamma_{1}\right)=N_{3} \theta_{2}(0)-\left[N_{0}+N_{3}\right] \theta_{3}(0)
\end{aligned}
$$

where $\forall n \in\{1,2,3\} \quad \lambda_{n}^{\prime \prime}=\frac{1}{N_{3} C_{r 3}}\left[\lambda_{n}{ }^{2}+\left(N_{1} C_{r 1}+N_{3} C_{r 3}\right) \lambda_{n}+N_{1}{ }^{2} C_{r 1}\right]$, and $K_{0}=\frac{-N_{1}{ }^{2} C_{r 1}}{N_{3} C_{r 3}}$.

$\underline{\text { Fluid } 3}$

$$
\theta_{3}(0)=\alpha_{3}+\beta_{3}+\gamma_{3}
$$

$$
\begin{gathered}
\left.\frac{d \theta_{3}}{d X}\right|_{X=0}=\alpha_{3} \lambda_{1}+\beta_{3} \lambda_{2}+\gamma_{3} \lambda_{3}=N_{3} \theta_{2}(0)-\left[N_{0}+N_{3}\right] \theta_{3}(0) \\
\frac{d^{2} \theta_{3}}{d X^{2}}+\left.\left[N_{0}+N_{3}\right] \frac{d \theta_{3}}{d X}\right|_{X=0}=\alpha_{3} \lambda_{1} \lambda_{1}^{\prime}+\beta_{3} \lambda_{2} \lambda_{2}^{\prime}+\gamma_{3} \lambda_{3} \lambda_{3}^{\prime}=N_{3}\left[N_{1} C_{r 1} \theta_{1}(0)-\left(N_{1} C_{r 1}+N_{3} C_{r 3}\right) \theta_{2}(0)+N_{3} C_{r 3} \theta_{3}(0)\right]
\end{gathered}
$$

where $\forall n \in\{1,2,3\} \lambda_{n}^{\prime}=\lambda_{n}+N_{0}+N_{3}$.

After some manipulations combining equations, coefficients $\alpha_{n}, \beta_{n}$ and $\gamma_{n}$ and solutions $\theta_{n}(X)$, for $n=1,2$ and 3 , can be expressed as functions of the unknown $\theta_{1}(0)$ :

$$
\begin{gathered}
\alpha_{n}=F_{n}^{\prime \prime} \theta_{1}(0)+K_{n}^{\prime \prime} \\
\beta_{n}=F_{n}^{\prime} \theta_{1}(0)+K_{n}^{\prime} \\
\gamma_{n}=F_{n} \theta_{1}(0)+K_{n} \\
\forall n \in\{1,2,3\} \quad \theta_{n}(X)=\left[F_{n}^{\prime \prime} e^{\lambda_{1} X}+F_{n}^{\prime} e^{\lambda_{2} X}+F_{n} e^{\lambda_{3} X}\right] \theta_{1}(0)+\left[K_{n}^{\prime \prime} e^{\lambda_{1} X}+K_{n}^{\prime} e^{\lambda_{2} X}+K_{n} e^{\lambda_{3} X}\right]
\end{gathered}
$$

where $F_{n}, F_{n}{ }^{\prime}, F_{n}{ }^{\prime \prime}, K_{n}, K_{n}{ }^{\prime}$ and $K_{n}{ }^{\prime \prime}$, for $n=1,2$ and 3, are coefficients which can be expressed in terms of $N_{0}, N_{1}, N_{3}, C_{r 1}, C_{r 3}, \lambda_{1}, \lambda_{2}, \lambda_{3}, \theta_{1}(1), \theta_{2}(0)$ and $\theta_{3}(0)$ (cf. Appendix).

Applying $\theta_{1}(X)$ and $\theta_{2}(X)$ from Equation (39) to the right-hand side of Equation (17) and through the integration of $d \theta_{1}(X) / d X$ from 0 to any $X$ and then from 1 to any $X$, yields:

0 to $X$

$$
\begin{aligned}
\theta_{1}(X)= & {\left[1+\xi_{1}\left(e^{\lambda_{1} X}-1\right)+\xi_{2}\left(e^{\lambda_{2} X}-1\right)+\xi_{3}\left(e^{\lambda_{3} X}-1\right)\right] \theta_{1}(0) } \\
& +\xi_{4}\left(e^{\lambda_{1} X}-1\right)+\xi_{5}\left(e^{\lambda_{2} X}-1\right)+\xi_{6}\left(e^{\lambda_{3} X}-1\right)
\end{aligned}
$$

1 to $X$

$$
\begin{aligned}
\theta_{1}(X)= & {\left[\xi_{1}\left(e^{\lambda_{1} X}-e^{\lambda_{1}}\right)+\xi_{2}\left(e^{\lambda_{2} X}-e^{\lambda_{2}}\right)+\xi_{3}\left(e^{\lambda_{3} X}-e^{\lambda_{3}}\right)\right] \theta_{1}(0) } \\
& +\xi_{4}\left(e^{\lambda_{1} X}-e^{\lambda_{1}}\right)+\xi_{5}\left(e^{\lambda_{2} X}-e^{\lambda_{2}}\right)+\xi_{6}\left(e^{\lambda_{3} X}-e^{\lambda_{3}}\right)+\theta_{1}(1)
\end{aligned}
$$

where $\xi_{1}, \xi_{2}, \xi_{3}, \xi_{4}, \xi_{5}$ and $\xi_{6}$ are coefficients which can be expressed in terms of $\lambda_{1}, \lambda_{2}, \lambda_{3}, N_{1}, F_{n}, F_{n}{ }^{\prime}$, $F_{n}{ }^{\prime \prime}, K_{n}, K_{n}{ }^{\prime}$ and $K_{n}{ }^{\prime \prime}$, for $n=1$ and 2 (cf. Appendix).

Equations (40) and (41) yield the following expression for the unknown $\theta_{1}(0)$ :

$$
\theta_{1}(0)=\frac{\xi_{4}\left(1-e^{\lambda_{1}}\right)+\xi_{5}\left(1-e^{\lambda_{2}}\right)+\xi_{6}\left(1-e^{\lambda_{3}}\right)+\theta_{1}(1)}{1+\xi_{1}\left(e^{\lambda_{1}}-1\right)+\xi_{2}\left(e^{\lambda_{2}}-1\right)+\xi_{3}\left(e^{\lambda_{3}}-1\right)}
$$


Thus, for $n=1,2$ and 3, the coefficients $\left(\alpha_{n}, \beta_{n}, \gamma_{n}\right)$ and the solutions $\theta_{n}(X)$ can be completely determined for any $X$ value between 0 and 1 . Consequently, the bulk temperatures $T_{n}(X)$ can be expressed as:

$$
\forall X \in\{0 \ldots 1\} \text { and } \forall n \in\{1,2,3\} \quad T_{n}(X)=T_{0}+\Delta T_{\text {in }} \theta_{n}(X)
$$

Outlet temperatures of the three fluids are computed from:

$$
\begin{aligned}
& T_{1 \text { out }}=T_{1}(0)=T_{0}+\Delta T_{\text {in }} \theta_{1}(0) \\
& T_{2 \text { out }}=T_{2}(1)=T_{0}+\Delta T_{\text {in }} \theta_{2}(1) \\
& T_{3 \text { out }}=T_{3}(1)=T_{0}+\Delta T_{\text {in }} \theta_{3}(1)
\end{aligned}
$$

The heat rate $\phi_{30(c)}$ transferred across the non-adiabatic outside surface can be now determined through the integration of $\theta_{3}(X)$ from 0 to 1 :

$$
\begin{gathered}
\phi_{30(c)}=U_{c} A_{c} \Delta T_{\text {in }} \int_{0}^{1} \theta_{3}(X) d X \\
\phi_{30(c)}=U_{c} A_{c} \Delta T_{\text {in }}\left[\frac{\alpha_{3}}{\lambda_{1}}\left(e^{\lambda_{1}}-1\right)+\frac{\beta_{3}}{\lambda_{2}}\left(e^{\lambda_{2}}-1\right)+\frac{\gamma_{3}}{\lambda_{3}}\left(e^{\lambda_{3}}-1\right)\right]
\end{gathered}
$$

For $n=1,2$ and 3, the heat flux $\phi_{n}$ assigned or retrieved by the fluid $n$ is computed from:

$$
\forall n \in\{1,2,3\} \quad \phi_{n}=C_{n}\left(T_{\text {out }}-T_{\text {in }}\right)_{n}
$$

\subsection{Effectiveness Relation}

Considering an analogy with a two-fluid heat exchanger, the overall effectiveness $E$ of a three-fluid heat exchanger is defined [26] as the ratio of the actual heat transfer rate $\phi_{\text {actual }}$ to the maximum possible heat transfer rate $\phi_{\max }$ :

$$
E=\frac{\phi_{\text {actual }}}{\phi_{\max }}
$$

As recommended by Sekulic and Kmecko [29], one should consider the particular purpose of the device to address the effectiveness issue for a three-fluid heat exchanger. As such, in this study, the actual heat transfer rate, $\phi_{\text {actual, }}$, is recovered by the fluid 2 :

$$
\phi_{\text {actual }}=\phi_{2}=C_{2}\left(T_{2 \text { out }}-T_{2 \text { in }}\right)
$$

Then, the maximum possible heat transfer rate, $\phi_{\max }$, that could be recovered by the fluid 2 , from both fluids 1 and 3, can be expressed as:

$$
\phi_{\max }=C_{\min (1 ; 2)} \Delta T_{\max (1 ; 2)}+C_{\min (3 ; 2)} \Delta T_{\max (3 ; 2)}
$$

where $\forall(i ; j) \in\{1 ; 2 ; 3\}^{2} / i \neq j, C_{\min (i ; j)}=\min \left(C_{i} ; C_{j}\right), \Delta T_{\max (i ; j)}=\max \left(T_{i \mathrm{in}} ; T_{j \text { in }}\right)-\min \left(T_{i \mathrm{in}} ; T_{j \mathrm{in}}\right)$.

It is worth mentioning that owing to the fluids properties considered in this study [8], in any case $C_{2}>C_{1}, C_{2}>C_{3}$ and $C_{1}>C_{3}$. It results that the maximum possible heat transfer rate $\phi_{\max }$ is given by:

$$
\phi_{\max }=C_{1}\left(T_{1 \text { in }}-T_{2 \text { in }}\right)+C_{3}\left(T_{3 \text { in }}-T_{2 \text { in }}\right)
$$


Thus, heat exchanger effectiveness $E$ is computed from:

$$
E=\frac{C_{2}\left(T_{2 \text { out }}-T_{2 \text { in }}\right)}{C_{1}\left(T_{1 \text { in }}-T_{2 \text { in }}\right)+C_{3}\left(T_{3 \text { in }}-T_{2 \text { in }}\right)}
$$

Using previous notations, the heat exchanger effectiveness $E$ can thus be written in the following form:

$$
E=\frac{\theta_{2}(1)-\theta_{2}(0)}{C_{r 1}+C_{r 3}}
$$

Since in any case $C_{2}>C_{1}+C_{3}$, the non-dimensional parameter $z$ defined as the ratio of the minimum heat capacity rate to the maximum heat capacity rate can be introduced. Hence:

$$
E=\frac{\theta_{2}(1)-\theta_{2}(0)}{z}
$$

with:

$$
z=C_{\min } / C_{\max }=\left(C_{1}+C_{3}\right) / C_{2}=C_{r 1}+C_{r 3}
$$

Finally, the heat exchanger effectiveness $E$ only depends on the non-dimensional parameter $z$ and the temperature ratio $\left(T_{2 \text { out }}-T_{2 \text { in }}\right) / \Delta T_{\text {in }}$ :

$$
E=\frac{T_{2 \text { out }}-T_{2 \text { in }}}{z \Delta T_{\text {in }}}
$$

\section{Parametric Study}

\subsection{Original Set of Input Data and Results}

The input data used in the parametric study are given in Table 1 . The diameters of the three tubes are 80,180 and $230 \mathrm{~mm}$ respectively and the total length is $1.5 \mathrm{~m}$. These values are close to measurements carried out on a bench test which is described in [8] and the comparison between experimental and numerical results showed that heat transfer rates are predicted with a relative difference lower than $5 \%$.

Table 1. Input data and outlet temperatures for both TCTHE-NI and TCTHE-PI models $(L=1.5 \mathrm{~m})$.

\begin{tabular}{cllccc}
\hline \multirow{2}{*}{$\boldsymbol{L}=\mathbf{1 . 5} \mathbf{~ m}$} & \multirow{2}{*}{$\boldsymbol{T}_{\mathbf{0}}=\mathbf{2 5}^{\circ} \mathbf{C}$} & Fluid 1 & Fluid 2 & Fluid 3 \\
\cline { 4 - 6 } & & FG & VA & CA \\
\hline \multirow{3}{*}{ Input data } & $D_{\mathrm{a}, \mathrm{b}, \mathrm{c}}$ & $(\mathrm{mm})$ & 80 & 180 & 230 \\
& $T_{\text {in } 1,2,3}$ & $\left({ }^{\circ} \mathrm{C}\right)$ & 180 & 15 & 60 \\
& $C_{1,2,3}$ & $(\mathrm{~W} / \mathrm{K})$ & 10 & 20 & 5 \\
\hline Computed data & $U_{\mathrm{a}, \mathrm{b}, \mathrm{c}}$ & $\left(\mathrm{W} /\left(\mathrm{m}^{2} \mathrm{~K}\right)\right)$ & 5.0 & 3.2 & 2.0 \\
\hline TCTHE-NI & $T_{\text {out } 1,2,3}$ & $\left({ }^{\circ} \mathrm{C}\right)$ & 153.09 & 31.6 & 38.2 \\
\hline TCTHE-PI & $T_{\text {out } 1,2,3}$ & $\left({ }^{\circ} \mathrm{C}\right)$ & 153.13 & 32.1 & 45.2 \\
\hline
\end{tabular}


The overall heat transfer coefficients $U$ of the three tubes $a, b$, and $c$ are calculated as explained previously in this paper. In case of perfect insulation, the parameter $U_{\mathrm{c}}$ is equal to zero. The last two rows of Table 1 give outlet temperatures obtained first by using the TCTHE model with no insulation (NI) which is presented in this paper and then by using the TCTHE with perfect insulation (PI) which has been developed by Ünal [23]. It can be noticed that inlet temperature of the combustion air (fluid 3) is higher than inlet temperature of the ventilation air (fluid 2) because the combustion air is preheated by the flue gases.

The heat capacity rate values, noted $\mathrm{C}$, correspond to flow rates of about 35,130 and $15 \mathrm{~m}^{3} / \mathrm{h}$ for the flue gases, the ventilation air and the combustion air respectively. These values were measured during laboratory tests and ventilation air flow rate meets the regulatory requirements of the ventilation of dwellings in France. Numerical calculations have been carried out for both TCTHE-NI and PI models to predict the temperature distributions of the three fluids along the heat exchanger (Figure 5). Considering the values adopted as input, the difference between temperature distribution of the TCTHE-NI and TCTHE-PI models does not appear very clearly except for the fluid 3 which is combustion air. However, even if the results obtained with the two models differ only at the second decimal for the flue gases, outlet temperature of each fluid is actually affected when taking into account the heat transfer with the outside, as it is displayed in Table 1.

Figure 5. Temperature distribution along the TCTHE-NI and PI $(L=1.5 \mathrm{~m})$.

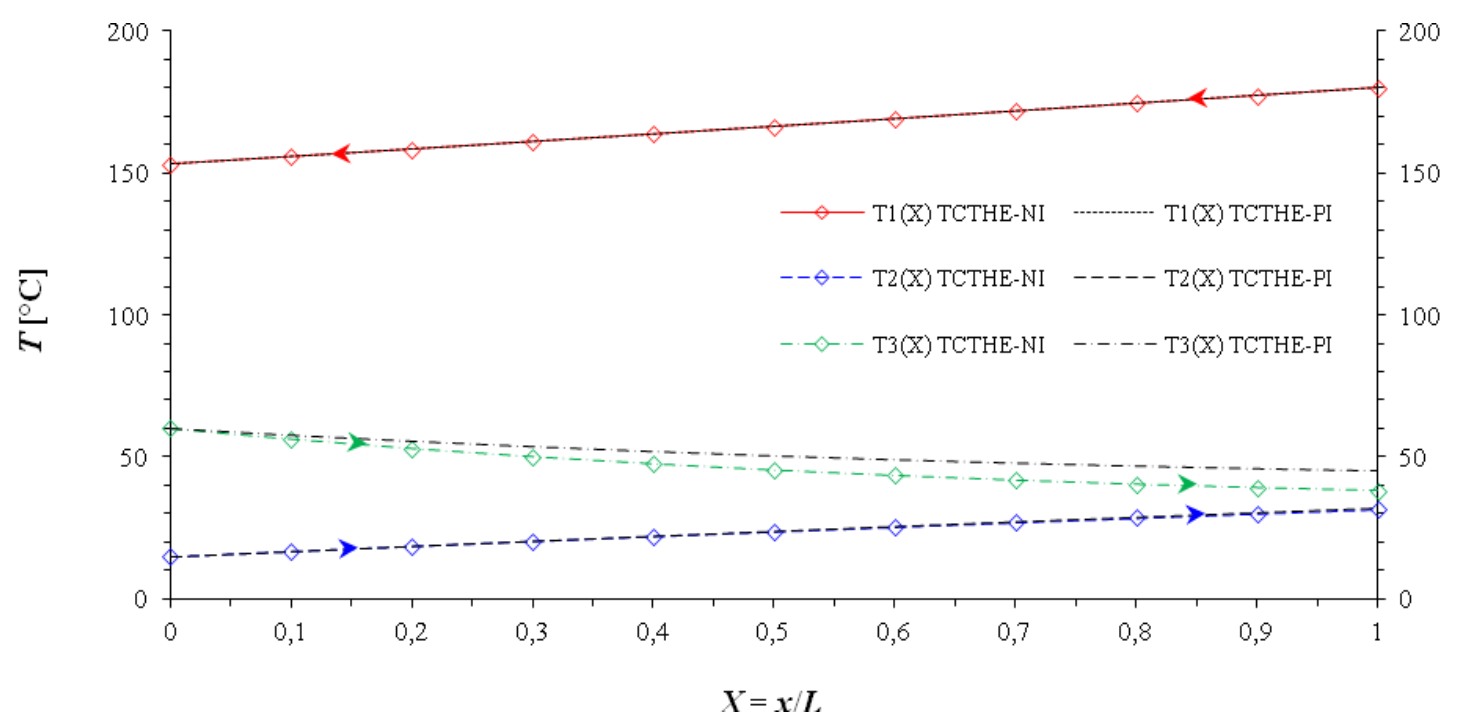

Then, the three graphs in Figure 6 show the heat balance for the TCTHE-NI $\left(U_{\mathrm{c}} \neq 0\right)$, TCTHE-NI $\left(U_{\mathrm{c}}=0\right)$ and TCTHE-PI models. Each graph totalizes on its left part the heat flux assigned by the flue gases and by the combustion air, and on its right part the heat flux retrieved by the ventilation air and by the outside in case of no insulation. As expected, the algebraic sum of $\phi_{\mathrm{OUT}}, \phi_{\mathrm{FG}}, \phi_{\mathrm{VA}}$ and $\phi_{\mathrm{CA}}$ is always equal to zero. Thus, the heat balance is verified for all models studied. Moreover, if the overall heat transfer coefficient of the external wall tube is taken very close to zero, like here with $U_{\mathrm{c}}=1.10^{-10} \mathrm{~W} /\left(\mathrm{m}^{2} \mathrm{~K}\right)$ for failing to divide by zero and lead to errors in the code, it must be emphasized that results of the TCTHE-NI $\left(U_{\mathrm{c}}=0\right)$ and TCTHE-PI models are in perfect agreements. 
Figure 6. Heat balance for the three TCTHE models $(L=1.5 \mathrm{~m})$.
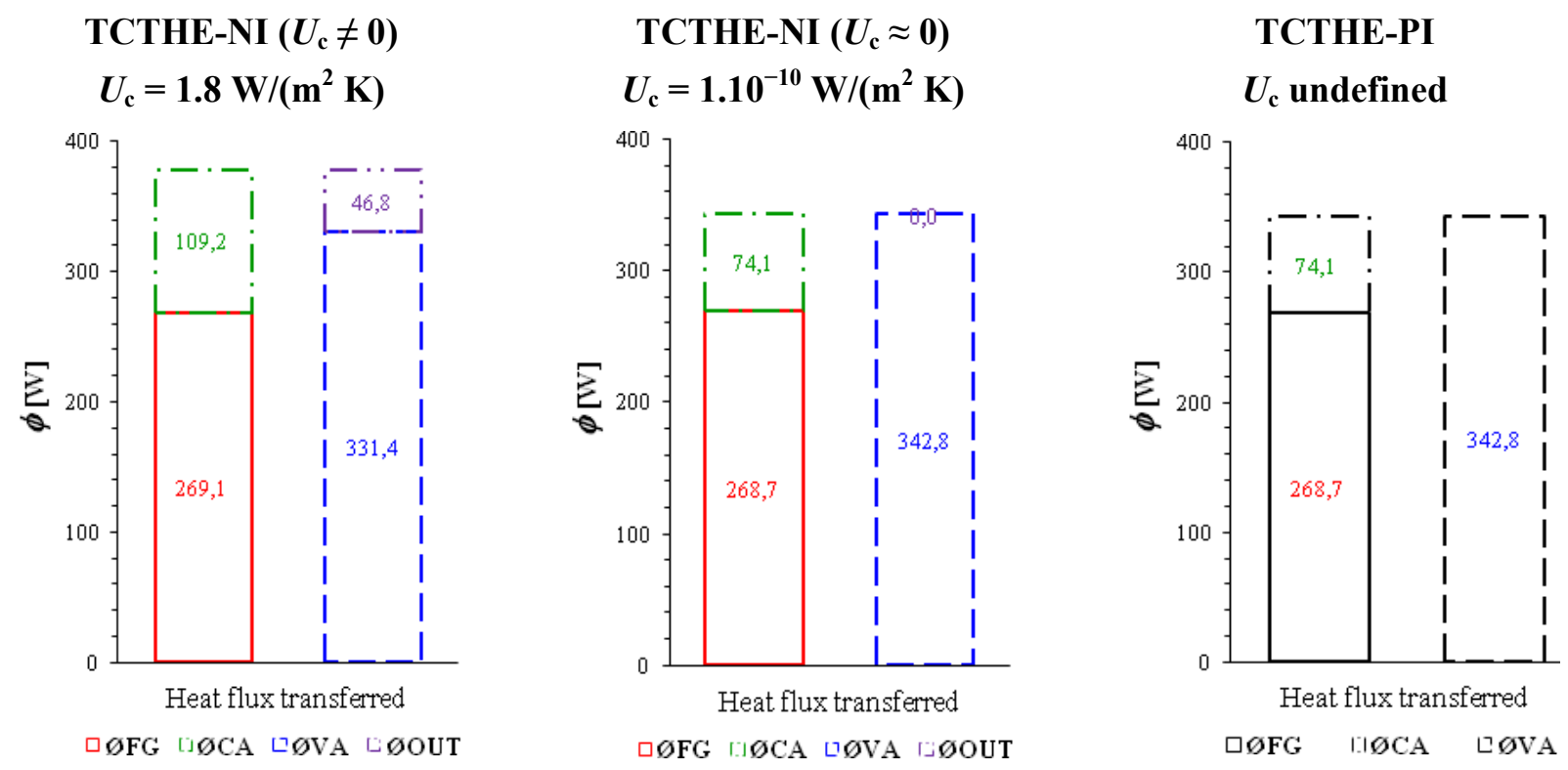

Although heat losses to the surroundings represent about $12 \%$ of the total heat transferred in the case of the TCTHE-NI, it is worth mentioning that the heat recovered by the ventilation air is only about 3\% lower than in the case of the TCTHE-PI. Indeed and as expected, it is the amount of heat assigned by the combustion air to the surroundings which is mainly affected depending on whether the heat exchanger is insulated from the outside or not. Thus, as this study is conducted with an industrial approach, adding a fourth tube containing thermal insulation is not a relevant solution to increase the heat recovered by the ventilation air. In addition, efficiency of the pellet stove is not degraded because the combustion air temperature increases highly between the outlet of the heat exchanger and the inlet of the stove. Indeed, just behind the appliance, the combustion air recovers heat directly from flue gases in the concentric tube used for connecting the chimney with the stove.

As the amount of heat recovered by the ventilation air is quite similar for both models, as well as there are identical values for $\phi_{\max }=1875 \mathrm{~W}$ and $z=0.75$, there is little difference between the performances of the two models. Exact values for effectiveness are $17.7 \%$ for the TCTHE-NI and $18.3 \%$ for the TCTHE-PI. Nevertheless, even if effectiveness values are quite low, the most important issues under consideration in this study are the fact that enough heat can be recovered by the ventilation air and the fact that the evacuation of flue gases is correctly maintained.

\subsection{Influence of Increasing Length}

It should also be emphasized that the temperature distribution appears to be quasi-linear along the exchanger on the graph in Figure 5, whereas the temperature expression is written in the form a sum of exponentials in Equation (22). However, the exponential evolution of the temperature distribution in the exchanger is stressed when increasing the tube length, as is it shown for example on the graph in Figure 7 where both TCTHE-NI and PI results are presented for the same input data as used previously, except for the length $L=10 \mathrm{~m}$. 
Figure 7. Temperature distribution along the TCTHE-NI and PI $(L=10 \mathrm{~m})$.

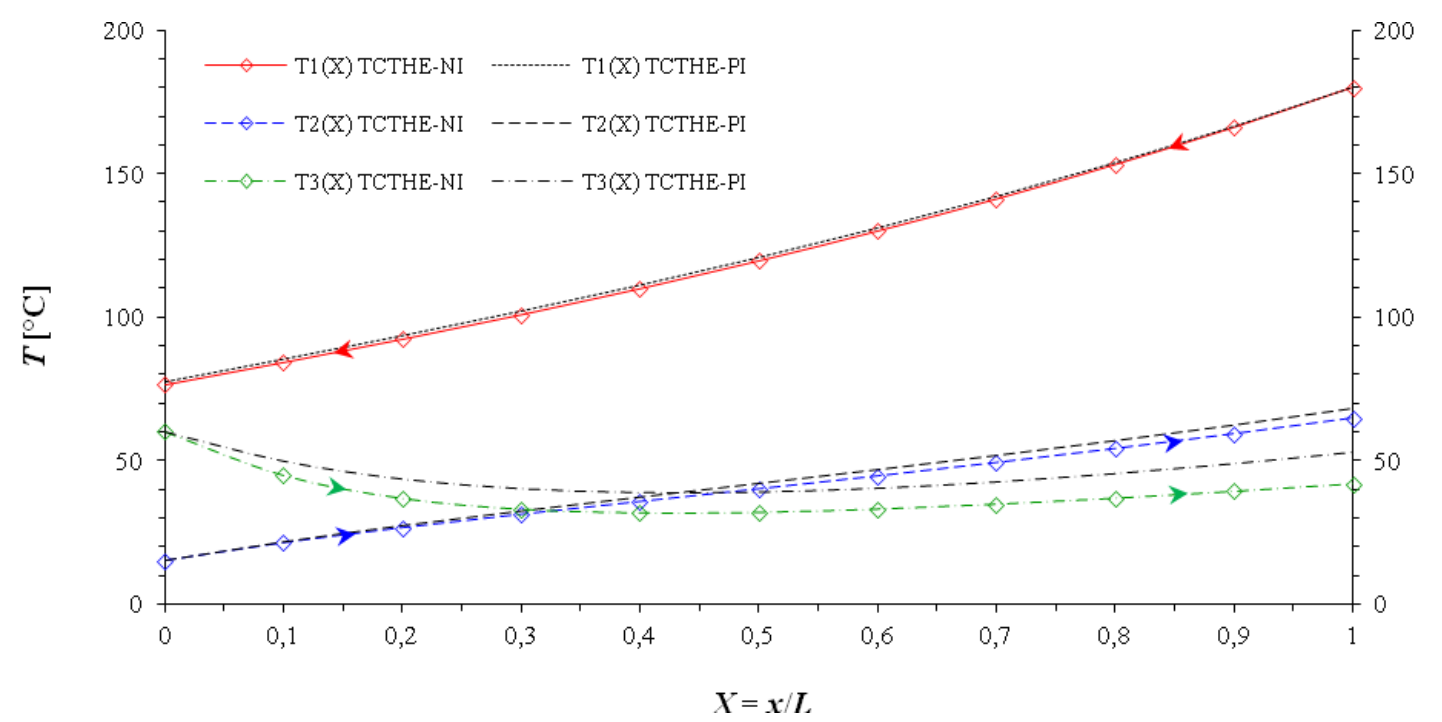

On the graph in Figure 7 at $X=1$, it should be noted that the outlet temperature of the combustion air (hot fluid 3) is lower than the outlet temperature of the ventilation air (cold fluid 2). This temperature cross phenomenon appears for both TCTHE-NI and TCTHE-PI models and reflects the reversal of heat exchanges from a certain point in the heat exchanger. Differences between the outlet temperatures are also more significant whether the heat exchanger is insulated or not, as evidenced by the values given in Table 2 .

Of course, such a $10 \mathrm{~m}$ length heat exchanger is not really appropriate for the combined system studied here. However, it should be stressed that in this case again, heat losses to the surroundings represent about $12 \%$ of the total heat transferred by both the flue gases and the combustion air. Here, as the maximum heat transfer rate is still the same, $\phi_{\max }=1875 \mathrm{~W}$, the effectiveness of the triple concentric tube heat exchanger reaches $53.0 \%$ if there is no insulation from the surroundings and $56.6 \%$ when perfectly insulated from the outside.

Table 2. Outlet temperatures for TCTHE-NI and TCTHE-PI models ( $L=10 \mathrm{~m})$.

\begin{tabular}{cllccc}
\hline \multirow{2}{*}{$\boldsymbol{L}=\mathbf{1 0} \mathbf{~ m}$} & \multirow{2}{*}{$\boldsymbol{T}_{\mathbf{0}}=\mathbf{2 5}^{\circ} \mathbf{C}$} & Fluid 1 & Fluid 2 & Fluid 3 \\
\cline { 3 - 6 } & & & FG & VA & CA \\
\hline Computed data & $U_{\mathrm{a}, \mathrm{b}, \mathrm{c}}$ & $\left(\mathrm{W} /\left(\mathrm{m}^{2} \mathrm{~K}\right)\right)$ & 5.0 & 2.5 & 1.5 \\
TCTHE-NI & $T_{\text {out } 1,2,3}$ & $\left({ }^{\circ} \mathrm{C}\right)$ & 76.5 & 64.7 & 42.0 \\
TCTHE-PI & $T_{\text {out } 1,2,3}$ & $\left({ }^{\circ} \mathrm{C}\right)$ & 77.5 & 68.0 & 53.0 \\
\hline
\end{tabular}

\subsection{Influence of Adding Fins}

In this study, fins could be added on the external wall of the inner tube to increase the amount of heat recovered by the ventilation air on the flue gas side. Considering the original set of input data for the TCTHE-NI model, the thickness, width and length of fins are $0.4 \mathrm{~mm}, 45 \mathrm{~mm}$ and $1160 \mathrm{~mm}$, respectively. The fins are located in the longitudinal direction of the flow and their volume represent less than $2 \%$ of the total ventilation air flow volume. So, we consider initially the flow is not affected significantly by adding fins. 
Several values of $C_{2}$ are considered to reflect the various flow rates of the ventilation air that may be encountered in the heat exchanger, i.e., parameter $z=0.75$ to 0.375 in experiments. As expected, the graph in Figure 8 shows that adding fins increases the exchanger effectiveness, but this trend is less pronounced when more than ten fins are added. As a result of this analysis, eight fins are planned to be added to the external wall of the inner tube to enhance the exchanger effectiveness without involving excessive manufacturing costs.

Figure 8. Effectiveness as a function of the number of fins for the TCTHE-NI model with the original set of input data and with ventilation air flow variations.

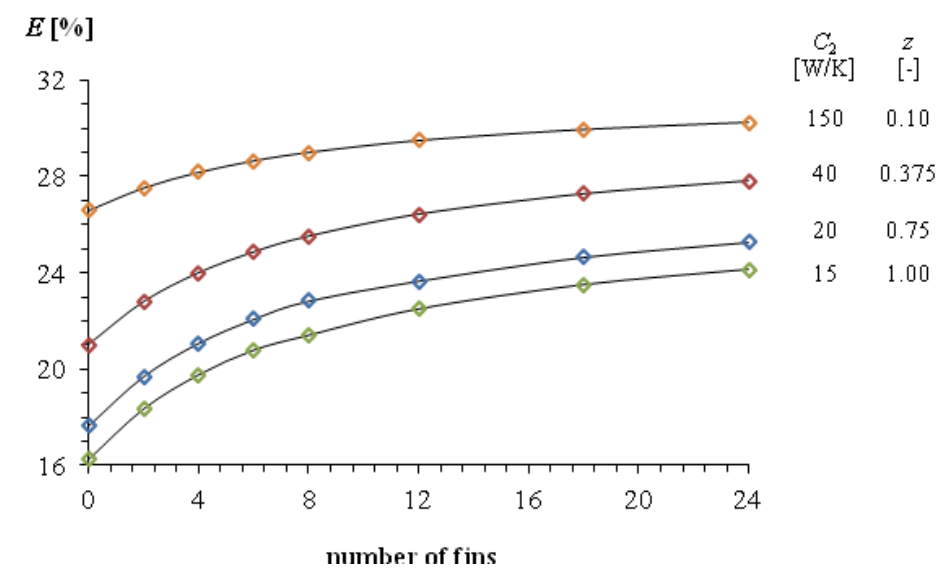

\subsection{Effectiveness Charts}

Considering the original set of input data presented in Table 1, a short parametric study is carried out to assess how the effectiveness $E$ of the TCTHE-NI is influenced by the four following input parameters: $C_{2}, D_{\mathrm{b}}, T_{2}$ in and $T_{0}$. Thus, the four graphs in Figure 9 show the variation of the effectiveness with one of the four previous parameters and with the number of transfer unit (NTU), which is a non-dimensional parameter defined here with previous notations:

$$
\mathrm{NTU}=\frac{U_{a} A_{a}+U_{b} A_{b}}{C_{1}+C_{3}}
$$

For each graph, several values of NTU are obtained by varying the length of the heat exchanger between 1 and $30 \mathrm{~m}$. However, it is worth noting that the NTU value is always lower than 0.5 when calculations are carried out with the original set of input data defined in this study.

First, Figure 9a shows that the effectiveness increases with the growth of the heat capacity rate $C_{2}$. Indeed, when the mass flow rate of the fluid2increases, itcausesbotha decrease of the non-dimensional parameter $z$ defined in Equation (57), and an increase of convective heat transfer coefficients $h_{2}$ int and $h_{2 \text { ext }}$ in the first annulus. As in the case of two-fluid heat exchangers, the effectiveness value is minimum for $z=1$ and maximum for $z \rightarrow 0$. Finally, the variation of $C_{2}$ has a fairly significant influence on the effectiveness, even for low values of NTU. 
Figure 9. Effectiveness charts for the TCTHE-NI model.

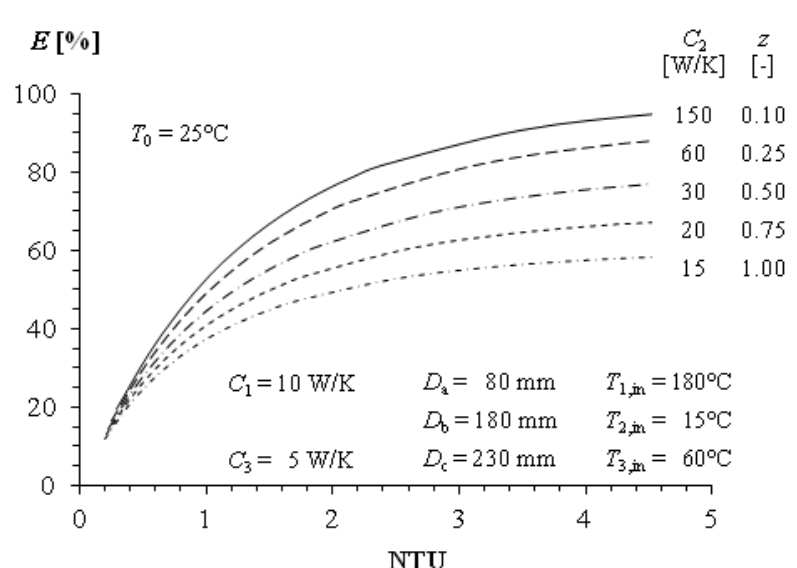

(a) Effectiveness variation with $C_{2}$

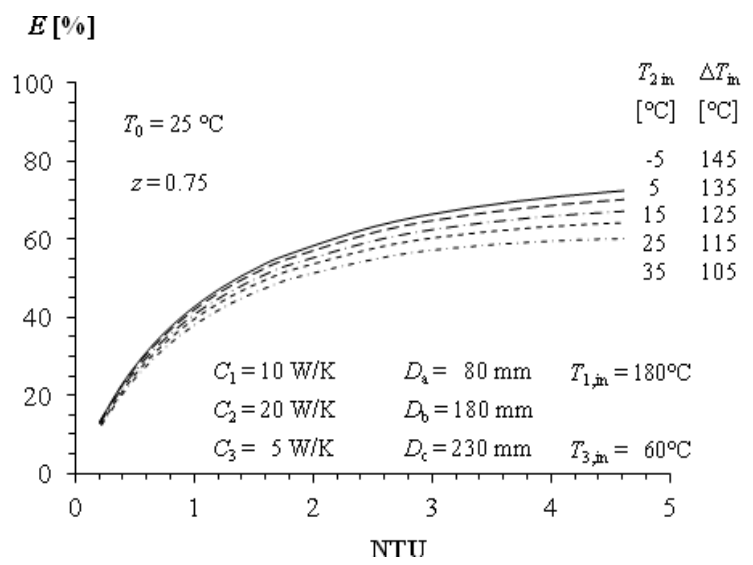

(c) Effectiveness variation with $T_{2}$ in

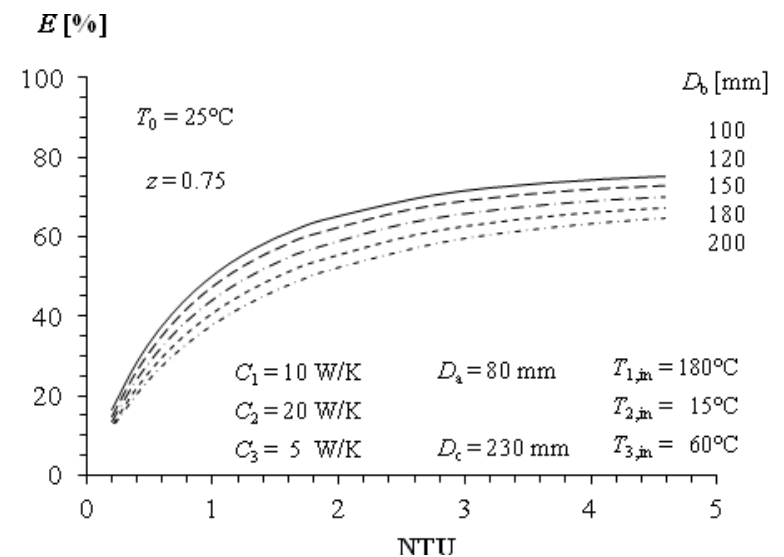

(b) Effectiveness variation with $D_{\mathrm{b}}$

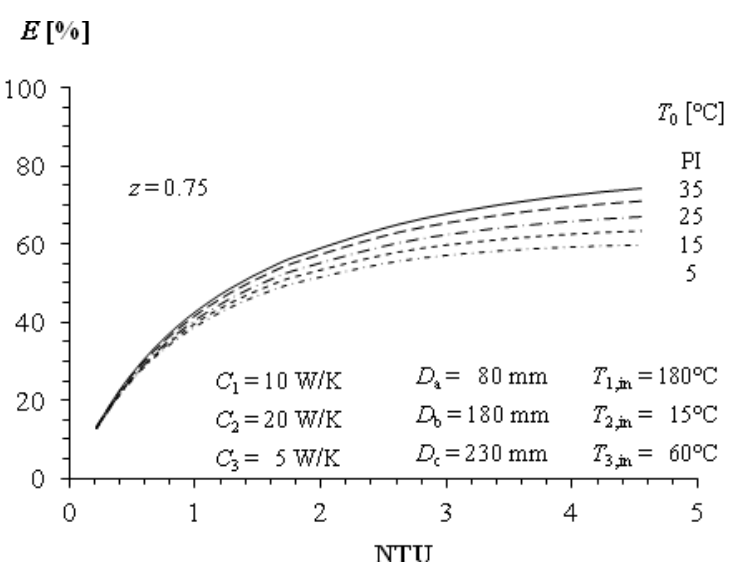

(d) Effectiveness variation with $T_{0}$

Then, as shown in Figure 9b, the effectiveness increases when the tube diameter $D_{\mathrm{b}}$ decreases. In simple words, reducing the flow area for the fluid 2 results in a significant increase of the convective heat transfer coefficients $h_{2}$ int and $h_{2}$ ext in the first annulus and this effect overrides the negative effect of the decrease in heat transfer surface area with fluid 3. Here again, the choice of the size of $D_{\mathrm{b}}$ has a significant influence on the effectiveness, even for low values of NTU and this observation is consistent with the findings of Ünal [24], which highlight that the relative sizes of the three tube diameters are important parameters that influence the performance of the exchanger.

Figure 9c shows that the effectiveness increases when the inlet temperature $T_{2}$ in decreases, i.e., when the non-dimensional parameter $\Delta T_{\text {in }}$ increases. Considering the specific combination of the counter-current co-current flow arrangement studied here, the actual heat recovered by the fluid 2 increases at a greater rate than the maximum heat recoverable. Nevertheless, it is of some interest to note that the variation of $T_{2}$ in has a relatively limited influence on the effectiveness, especially for low values of NTU.

When the external temperature $T_{0}$ increases, Figure $9 \mathrm{~d}$ shows that the effectiveness of the exchange also increases. In addition and as expected, the effectiveness reaches its maximum when the TCTHE is perfectly isolated (PI). However, as mentioned in the previous sections, the variation of the outside temperature mainly affects the outlet temperature of the combustion air. Thus, $T_{0}$ has little influence on the effectiveness for low NTU values, but its impact becomes more significant for higher NTU values. 
The analysis of the four graphs in Figure 9 verifies the consistency of the mathematical model presented in the first part of this study and the equations derived can be used to assess the performance of a TCTHE-NI. Then the effectiveness is revealed to be more significantly influenced by parameters $C_{2}$ or $D_{\mathrm{b}}$ than by parameters $T_{2}$ in or $T_{0}$.

\subsection{Importance of Heat Losses}

The heat transferred to the outside, $\phi_{\text {OUT }}$, may be compared to the total heat assigned or retrieved, $\phi_{\mathrm{TOT}}=\phi_{\mathrm{VA}}+\phi_{\mathrm{OUT}}=\phi_{\mathrm{FG}}+\phi_{\mathrm{CA}}$, in the TCTHE-NI. Figure10 shows the variations of the ratio $\phi_{\text {OUT }} / \phi_{\text {TOT }}$ with increasing NTU values. Of course, this ratio is always equal to zero in case of perfect insulation (PI). But depending on the surroundings temperature, $T_{0}$, heat losses to the outside may represent a significant part of heat transfer that occurs in the exchanger.

Considering the original set of input data, Figure 10a shows that heat losses remain below $20 \%$ for NTU $<1.5$ and $T_{0}>10{ }^{\circ} \mathrm{C}$, reflecting the majority of cases that may be encountered in our application. Indeed, in the case of the combined system studied here, the TCTHE-NI is commonly located just behind the heating appliance in a closet in which the temperature remains between $15{ }^{\circ} \mathrm{C}$ and $25{ }^{\circ} \mathrm{C}$.

Figure 10. Percentage of heat transferred to the surroundings for the TCTHE-NI model with the original set of input data (a) and with an increase of the ventilation air flow (b).

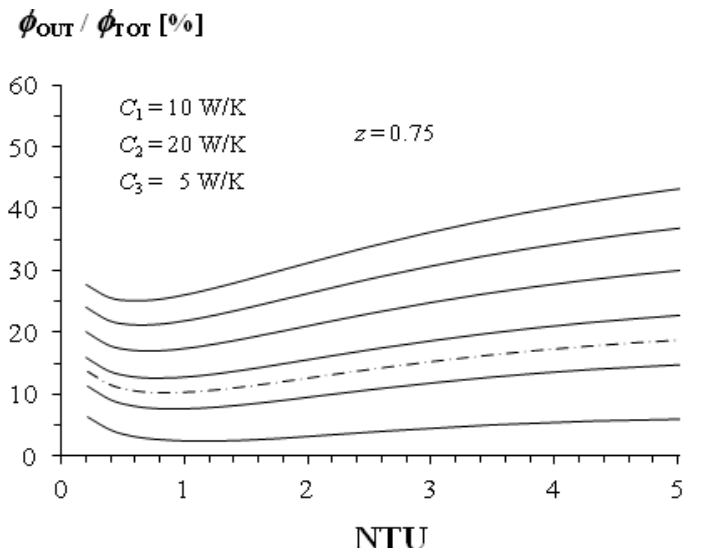

(a) Percentage of heat losses with $C_{2}=20 \mathrm{~W} / \mathrm{K}$

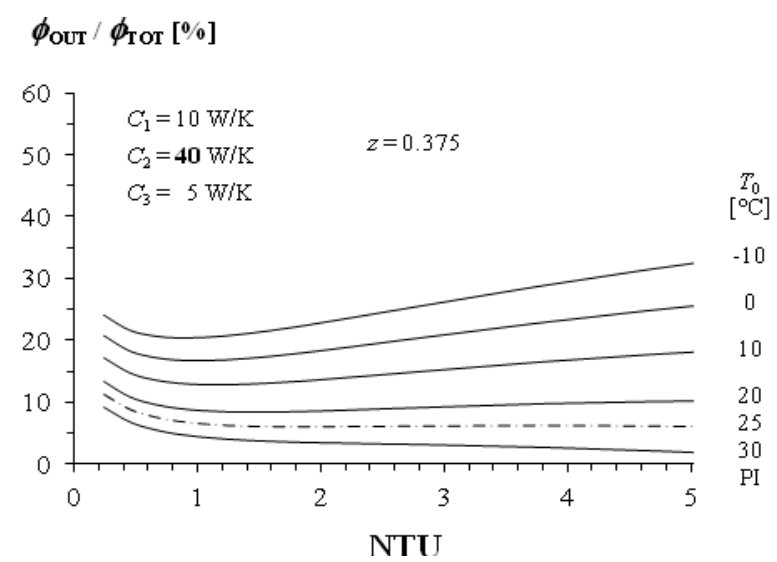

(b) Percentage of heat losses with $C_{2}=40 \mathrm{~W} / \mathrm{K}$

In addition, as shown in Figure 10b, heat transfers focus on the side of the ventilation air as soon as its flow rate increases. In the TCTHE-NI, the proportion of heat losses to the surroundings compared to the total heat transferred remains therefore lower than $10 \%$ if the ambient air temperature is at least $20{ }^{\circ} \mathrm{C}$. Hence, as heat losses to the outside remain moderate in our case study, adding a fourth tube containing insulation material cannot be justified in view of larger investment and manufacturing costs. In addition, heat losses from the heat exchanger are in the heated area. Therefore, it is not really heat losses.

\section{Conclusions}

Analytical solutions for the steady-state temperature of three heat exchanging fluids along the length of a triple concentric tube heat exchanger with no insulation at the outer surface are obtained for 
a specific combination of counter-current and co-current arrangement. The amount of heat transferred to the outside through the external tube is also calculated. For a case study, the temperature distribution of the three fluids is graphically represented and its exponential variation is stressed by increasing the length of the heat exchanger. Heat balance calculations and comparison with the perfectly insulated model have provided validation of the mathematical model developed in this paper. With the input data values of the parametric study conducted here, the temperature of the ventilation air increases by about $15{ }^{\circ} \mathrm{C}$ in the heat exchanger while the temperature of flue gases and combustion air decreases about $30{ }^{\circ} \mathrm{C}$ and $20{ }^{\circ} \mathrm{C}$ respectively. Then the importance of adding fins to the external surface of the inner tube of the TCTHE-NI is pointed out, with a potential increase of about $6 \%$ for eight fins, and the influence of several parameters, especially $C_{2}$ and $D_{\mathrm{b}}$, on the heat exchanger effectiveness is also assessed through a parametric study conducted in the continuity of the case study. Heat losses to the outside represent around $10 \%$ of the total heat transferred in the TCTHE-NI for the set of input data used in the case study, so insulation of the heat exchanger is not really required if it is placed in the heated volume of the dwelling.

Finally, this theoretical study could be extended to the various flow arrangements encountered in a triple concentric tube heat exchanger and the equations derived here could also be used to perform sizing calculations. In addition, we are aware that this model developed in this study is quite simple but comparison between experimental and numerical results showed that heat transfer rates are predicted with a relative difference lower than 5\% [8]. Furthermore, CFD approach will be used in a future work in order to study in more details the fluid flows within the heat exchanger.

\section{Acknowledgments}

This work was supported in part by the Poitou-Charentes Regional Council and by the Calyxis cluster of expertise in risk.

\section{References}

1. Plateforme de Recherche et D'expérimentation sur l'Energie dans le Bâtiment (PREBAT); International Building and Energy Comparison-Final Report; PREBAT: Paris, France, 2007.

2. Mardiana-Idayu, A.; Riffat, S.B. Review on heat recovery technologies for building applications. Energy Buildings 2012, 16, 1241-1255.

3. El Fouih, Y.; Stabat, P.; Rivière, P.; Hoang, P.; Archambault, V. Adequacy of air-to-air heat recovery ventilation system applied in low energy buildings. Energy Buildings 2012, 54, 29-39.

4. Laverge, J.; Janssens, A. Heat recovery ventilation operation traded off against natural and simple exhaust ventilation in Europe by primary energy factor, carbon dioxide emission, household consumer price and exergy. Energy Buildings 2012, 50, 315-323.

5. Feist, W.; Schnieders, J.; Dorer, V.; Haas, A. Re-inventing air heating: Convenient and comfortable within the frame of the Passive House concept. Energy Buildings 2005, 37, 1186-1203.

6. Pfluger, R. Wood Stoves in Passive Houses-Strategies for Comfort. In Proceedings of the 12th International Conference on Passive House, Nuremberg, Germany, 11-12 April 2008. 
7. Consumer Safety Commission. Recommendation on Hot Air Redistribution Systems. Available online: http://www.securiteconso.org/article230.html (assessed on 16 September 2012).

8. Peigné, P. Étude d'un système combiné de ventilation et de chauffage au bois dans les bâtiments à basse consommation d'énergie. Ph.D. Thesis, Université La Rochelle, La Rochelle, France, 2012.

9. Morley, T.B. Exchange of heat between three fluids. Engineer 1933, 155, 314-316.

10. Rabinovich, G.D. Steady state heat transfer among three streams in a parallel flow recuperator [in Russian]. Inzh.-Fiz. Zh. 1961, 4, 37-43.

11. Wolf, J. General solution of the equations of parallel flow multichannel heat exchangers. Int. J. Heat Mass Tran. 1964, 7, 901-919.

12. Aulds, D.D.; Barron, R.F. Three-fluid heat exchanger effectiveness. Int. J. Heat Mass Tran. 1967, 10, 1457-1462.

13. Sorlie, T. Three Fluid Heat Exchanger Design Theory: Counter and Parallel Flow; Department of Mechanical Engineering, Stanford University: Stanford, CA, USA, 1962.

14. Zuritz, C.A. On the design of triple concentric-tube heat exchangers. J. Food Process Eng. 1990, 12, 113-130.

15. Shpil'rain, E.E.; Yakimovich, K.A. Heat transfer between three media in triple countercurrent pipe flow. Inzh.-Fiz. Zh. 1982, 43, 1028-1033.

16. Shpil'rain, E.E.; Yakimovich, K.A. Heat exchange between three streams in pipes of variable cross section. J. Eng. Thermophys. 1983, 45, 937-939.

17. Krishnamurty, V.V.G.; Rao, C.V. Heat transfer in three-fluid heat exchangers. Indian J. Technol. 1964, 2, 325-327.

18. Krishnamurty, V.V.G. Heat transfer in multi-fluid heat exchangers. Indian J. Technol. 1966, 4, 167-169.

19. Sekulic, D.P. A compact solution of the parallel flow three-fluid heat exchanger problem. Int. J. Heat Mass Tran. 1994, 37, 2183-2187.

20. Sekulic, D.P.; Shah, R.K. Thermal design theory of three-fluid heat exchangers. Adv. Heat Tran. 1995, 26, 219-328.

21. Prasad, R.C. Analytical solution for a double-pipe heat exchanger with non-adiabatic condition at the outer surface. Int. Commun. Heat Mass. 1987, 14, 665-672.

22. Prasad, R.C. Generalized solution and effectiveness for concentric tube heat exchangers. Int. J. Heat Mass Tran. 1988, 31, 2571-2578.

23. Ünal, A. Theoretical analysis of triple concentric-tube heat exchangers, Part 1: Mathematical modelling. Int. Commun. Heat Mass. 1998, 25, 949-958.

24. Ünal, A. Theoretical analysis of triple concentric-tube heat exchangers, Part 2: Case studies. Int. Commun. Heat Mass. 2001, 28, 243-256.

25. Ünal, A. Effectiveness-NTU relations for triple concentric-tube heat exchangers. Int. Commun. Heat Mass. 2003, 30, 261-272.

26. Incropera, F.P.; DeWitt, D.P.; Bergman, T.L.; Lavine, A.S. Fundamentals of Heat and Mass Transfer, 6th ed.; Wiley: New York, NY, USA, 2006.

27. Jakob, M. Heat Transfer; John Wiley \& Sons: New York, NY, USA, 1949.

28. García-Valladares, O. Numerical simulation of triple concentric-tube heat exchangers. Int. J. Therm. Sci. 2004, 43, 979-991. 
29. Sekulic, D.P.; Kmecko, L. Three-fluid heat exchanger effectiveness. J. Heat Tran. 1995, 117, 226-227.

\section{Appendix: Explanation of the Mathematical Model Coefficients}

Coefficients $\alpha_{\mathrm{n}}, \beta_{\mathrm{n}}$ and $\gamma_{\mathrm{n}}$ are given by Equations (36)-(38):

$$
\forall n \in\{1,2,3\} \quad \alpha_{n}=F_{n}^{\prime \prime} \theta_{1}(0)+K_{n}^{\prime \prime} \quad \beta_{n}=F_{n}^{\prime} \theta_{1}(0)+K_{n}^{\prime} \quad \gamma_{n}=F_{n} \theta_{1}(0)+K_{n}
$$

where $F_{n}, F_{n}{ }^{\prime}, F_{n}{ }^{\prime \prime}, K_{n}, K_{n}{ }^{\prime}$ and $K_{n}{ }^{\prime \prime}$, for $n=1,2$ and 3, are coefficients which can be expressed in terms of $N_{0}, N_{1}, N_{3}, C_{r 1}, C_{r 3}, \lambda_{1}, \lambda_{2}, \lambda_{3}, \theta_{1}(1), \theta_{2}(0)$ and $\theta_{3}(0)$.

$n=1$

$$
\begin{array}{lll}
\alpha_{1}=F_{1}^{\prime \prime} \theta_{1}(0)+K_{1}^{\prime \prime} & K_{1}^{\prime \prime}=G_{1}^{\prime \prime} \theta_{2}(0)+H_{1}^{\prime \prime} \theta_{1}(1) & F_{1}^{\prime \prime}=1-\left(F_{1}^{\prime}+F_{1}\right) \\
\beta_{1}=F_{1}^{\prime} \theta_{1}(0)+K_{1}^{\prime} & K_{1}^{\prime}=G_{1}^{\prime} \theta_{2}(0)+H_{1}^{\prime} \theta_{1}(1) & G_{1}^{\prime \prime}=-\left(G_{1}^{\prime}+G_{1}\right) \\
\gamma_{1}=F_{1} \theta_{1}(0)+K_{1} & K_{1}=G_{1} \theta_{2}(0)+H_{1} \theta_{1}(1) & H_{1}^{\prime \prime}=-\left(H_{1}^{\prime}+H_{1}\right) \\
F_{1}^{\prime}=\frac{1}{\lambda_{2}-\lambda_{1}}\left[\left(N_{1}-\lambda_{1}\right)-F_{1}\left(\lambda_{3}-\lambda_{1}\right)\right] & F_{1}=-F\left[\left(\lambda_{2}-\lambda_{1}\right) e^{\lambda_{1}}+\left(e^{\lambda_{2}}-e^{\lambda_{1}}\right)\left(N_{1}-\lambda_{1}\right)\right] \\
G_{1}^{\prime}=\frac{-1}{\lambda_{2}-\lambda_{1}}\left[N_{1}+G_{1}\left(\lambda_{3}-\lambda_{1}\right)\right] & G_{1}=F\left(e^{\lambda_{2}}-e^{\lambda_{1}}\right) N_{1} & H_{1}=F\left(\lambda_{2}-\lambda_{1}\right) \\
H_{1}^{\prime}=\frac{-1}{\lambda_{2}-\lambda_{1}}\left[H_{1}\left(\lambda_{3}-\lambda_{1}\right)\right] & F=\left[\left(\lambda_{2}-\lambda_{1}\right)\left(e^{\lambda_{3}}-e^{\lambda_{1}}\right)-\left(\lambda_{3}-\lambda_{1}\right)\left(e^{\lambda_{2}}-e^{\lambda_{1}}\right)\right]^{-1}
\end{array}
$$

$n=2$

$$
\begin{array}{ll}
\alpha_{2}=F_{2}^{\prime \prime} \theta_{1}(0)+K_{2}^{\prime \prime} & K_{2}^{\prime \prime}=\theta_{2}(0)-\left(K_{2}^{\prime}+K_{2}\right) \\
\beta_{2}=F_{2}^{\prime} \theta_{1}(0)+K_{2}^{\prime} & K_{2}^{\prime}=\frac{-1}{\lambda_{2}-\lambda_{1}}\left[\left(N_{1} C_{r 1}+N_{3} C_{r 3}+\lambda_{1}\right) \theta_{2}(0)-N_{3} C_{r 3} \theta_{3}(0)+\left(\lambda_{3}-\lambda_{1}\right) K_{2}\right] \\
\gamma_{2}=F_{2} \theta_{1}(0)+K_{2} & K_{2}=-G\left[\frac{\left(N_{1} C_{r 1}+N_{3} C_{r 3}+\lambda_{1}\right)}{\lambda_{2}-\lambda_{1}}+\frac{\left(N_{3}-\lambda_{1}^{\prime \prime}\right)}{\lambda_{2}^{\prime \prime}-\lambda_{1}^{\prime \prime}}\right] \theta_{2}(0)+G\left[\frac{N_{3} C_{r 3}}{\lambda_{2}-\lambda_{1}}+\frac{N_{0}+N_{3}}{\lambda_{2}^{\prime \prime}-\lambda_{1}^{\prime \prime}}\right] \theta_{3}(0) \\
F_{2}^{\prime \prime}=-\left(F_{2}^{\prime}+F_{2}\right) & F_{2}^{\prime}=\frac{1}{\lambda_{2}-\lambda_{1}}\left[N_{1} C_{r 1}-F_{2}\left(\lambda_{3}-\lambda_{1}\right)\right] \quad G=\left[\frac{\lambda_{3}-\lambda_{1}}{\lambda_{2}-\lambda_{1}}-\frac{\lambda_{3}^{\prime \prime}-\lambda_{1}^{\prime \prime}}{\lambda_{2}^{\prime \prime}-\lambda_{1}^{\prime \prime}}\right]^{-1} \\
F_{2}=G\left[\frac{N_{1} C_{r 1}}{\lambda_{2}-\lambda_{1}}+\frac{K_{0}}{\lambda_{2}^{\prime \prime}-\lambda_{1}^{\prime \prime}}\right] \quad \text { with } F_{1}^{\prime \prime}+F_{1}^{\prime}+F_{1}=1 \quad
\end{array}
$$

$n=3$

$\alpha_{3}=F_{3}^{\prime \prime} \theta_{1}(0)+K_{3}^{\prime \prime}$

$$
K_{3}^{\prime \prime}=G_{3}^{\prime \prime} \theta_{2}(0)+H_{3}^{\prime \prime} \theta_{1}(1)
$$$$
F_{3}^{\prime \prime}=1-\left(F_{3}^{\prime}+F_{3}\right)
$$$$
\beta_{3}=F_{3}^{\prime} \theta_{1}(0)+K_{3}^{\prime}
$$$$
K_{3}^{\prime}=G_{3}^{\prime} \theta_{2}(0)+H_{3}^{\prime} \theta_{1}(1)
$$$$
G_{3}^{\prime \prime}=-\left(G_{3}^{\prime}+G_{3}\right)
$$

$\gamma_{3}=F_{3} \theta_{1}(0)+K_{3}$

$K_{3}=G_{3} \theta_{2}(0)+H_{3} \theta_{1}(1)$

$H_{3}^{\prime \prime}=-\left(H_{3}^{\prime}+H_{3}\right)$ 


$$
\begin{array}{ll}
F_{3}^{\prime}=\frac{-1}{\lambda_{2}-\lambda_{1}}\left[\left(N_{0}+N_{3}+\lambda_{1}\right)+F_{3}\left(\lambda_{3}-\lambda_{1}\right)\right] & F_{3}=H\left[\left(\lambda_{2}-\lambda_{1}\right)\left(N_{3}^{2} C_{r 3}-\lambda_{1} \lambda_{1}^{\prime}\right)+\left(\lambda_{2} \lambda_{2}^{\prime}-\lambda_{1} \lambda_{1}^{\prime}\right)\left(N_{0}+N_{3}+\lambda_{1}\right)\right] \\
G_{3}^{\prime}=\frac{1}{\lambda_{2}-\lambda_{1}}\left[N_{3}-G_{3}\left(\lambda_{3}-\lambda_{1}\right)\right] & G_{3}=-H N_{3}\left[\left(\lambda_{2} \lambda_{2}^{\prime}-\lambda_{1} \lambda_{1}^{\prime}\right)+\left(N_{1} C_{r 1}+N_{3} C_{r 3}\right)\left(\lambda_{2}-\lambda_{1}\right)\right] \\
H_{3}^{\prime}=\frac{-1}{\lambda_{2}-\lambda_{1}}\left[H_{3}\left(\lambda_{3}-\lambda_{1}\right)\right] & H_{3}=H\left(\lambda_{2}-\lambda_{1}\right) N_{3} N_{1} C_{r 1} \\
H=\left[\left(\lambda_{3} \lambda_{3}^{\prime}-\lambda_{1} \lambda_{1}^{\prime}\right)\left(\lambda_{2}-\lambda_{1}\right)-\left(\lambda_{3}-\lambda_{1}\right)\left(\lambda_{2} \lambda_{2}^{\prime}-\lambda_{1} \lambda_{1}^{\prime}\right)\right]^{-1}
\end{array}
$$

As presented in Equations (22) and (39), general solutions of the system of 3 linear homogeneous third order ordinary differential equations are in the form of a sum of exponential functions in $X$.

$\forall n \in\{1,2,3\}$

$$
\begin{aligned}
& \theta_{n}(X)=\alpha_{n} e^{\lambda_{1} X}+\beta_{n} e^{\lambda_{2} X}+\gamma_{n} e^{\lambda_{3} X} \\
& \theta_{n}(X)=\left[F_{n}^{\prime \prime} e^{\lambda_{1} X}+F_{n}^{\prime} e^{\lambda_{2} X}+F_{n} e^{\lambda_{3} X}\right] \theta_{1}(0)+\left[K_{n}^{\prime \prime} e^{\lambda_{1} X}+K_{n}^{\prime} e^{\lambda_{2} X}+K_{n} e^{\lambda_{3} X}\right]
\end{aligned}
$$

$\forall n \in\{1,2,3\}$

with

$$
\theta_{1}(0)=\frac{\xi_{4}\left(1-e^{\lambda_{1}}\right)+\xi_{5}\left(1-e^{\lambda_{2}}\right)+\xi_{6}\left(1-e^{\lambda_{3}}\right)+\theta_{1}(1)}{1+\xi_{1}\left(e^{\lambda_{1}}-1\right)+\xi_{2}\left(e^{\lambda_{2}}-1\right)+\xi_{3}\left(e^{\lambda_{3}}-1\right)}
$$

where $\xi_{1}, \xi_{2}, \xi_{3}, \xi_{4}, \xi_{5}$ and $\xi_{6}$ are coefficients which can be expressed in terms of $\lambda_{1}, \lambda_{2}, \lambda_{3}, N_{1}, F_{n}, F_{n}{ }^{\prime}$, $F_{n}{ }^{\prime \prime}, K_{n}, K_{n}{ }^{\prime}$ and $K_{n}{ }^{\prime \prime}$, for $n=1$ and 2 .
$\xi_{1}=\frac{N_{1}}{\lambda_{1}}\left(F_{1}^{\prime \prime}-F_{2}^{\prime \prime}\right)$
$\xi_{2}=\frac{N_{1}}{\lambda_{2}}\left(F_{1}^{\prime}-F_{2}^{\prime}\right)$
$\xi_{3}=\frac{N_{1}}{\lambda_{3}}\left(F_{1}-F_{2}\right)$
$\xi_{4}=\frac{N_{1}}{\lambda_{1}}\left(K_{1}^{\prime \prime}-K_{2}^{\prime \prime}\right)$
$\xi_{5}=\frac{N_{1}}{\lambda_{2}}\left(K_{1}^{\prime}-K_{2}^{\prime}\right)$
$\xi_{6}=\frac{N_{1}}{\lambda_{3}}\left(K_{1}-K_{2}\right)$

(C) 2013 by the authors; licensee MDPI, Basel, Switzerland. This article is an open access article distributed under the terms and conditions of the Creative Commons Attribution license (http://creativecommons.org/licenses/by/3.0/). 